\title{
Bacterial carotenoids suppress Caenorhabditis elegans surveillance and defense of translational dysfunction
}

\section{J. Amaranath Govindan ${ }^{1,2}$, Elamparithi Jayamani ${ }^{1,2}$, Victor Lelyveld ${ }^{3}$, Jack Szostak ${ }^{1,2,3}$, and Gary Ruvkun ${ }^{1,2,3}$}

1 Department of Molecular Biology, Massachusetts General Hospital, Boston, MA 02114 , USA,

2 Department of Genetics, Harvard Medical School, Boston, MA 02115, USA,

3 Center for Computational and Integrative Biology, Massachusetts General

Hospital, Boston, Massachusetts 02114, United States

Corresponding author: ruvkun@molbio.mgh.harvard.edu

December 30, 2019

\section{Abstract}

Microbial toxins and virulence factors often target the eukaryotic translation machinery. Caenorhabditis elegans surveils for such microbial attacks by monitoring translational competence, and if a deficit is detected, particular drug detoxification and bacterial defense genes are induced. The bacteria Kocuria rhizophila has evolved countermeasures to animal translational surveillance and defense pathways. Here, we used comprehensive genetic analysis of Kocuria rhizophila to identify the bacterial genetic pathways that inhibit C. elegans translational toxin surveillance and defense. Kocuria rhizophila mutations that disrupt its ability to disable animal immunity and defense map to multiple steps in the biosynthesis of a 50-carbon bacterial carotenoid from 5 carbon precursors. Extracts of the $\mathrm{C}_{50}$ carotenoid from wild type $K$. rhizophila could restore this bacterial anti-immunity activity to $K$. rhizophila carotenoid biosynthetic mutant. Corynebacterium glutamicum, also inhibits the $C$. elegans translation detoxification response by producing the $\mathrm{C}_{50}$ carotenoid decaprenoxanthin, and $C$. glutamicum carotenoid mutants are defective in this suppression of $C$. elegans detoxification. Consistent with the salience of these bacterial countermeasures to animal drug responses, bacterial carotenoids sensitize $C$. elegans to drugs that target translation and inhibit food aversion behaviors normally induced by protein translation toxins or mutations. The surveillance and response to toxins is mediated by signaling pathways conserved across 
animal phylogeny, suggesting that these bacterial carotenoids may also suppress such human immune and toxin responses.

\section{Introduction}

Many bacterial toxins and virulence factors target the highly conserved RNAs and proteins of the ribosome and associated translation factors. In response to such toxin attacks, eukaryotic defense responses include the induction of specific suites of drug detoxification and anti-bacterial immunity genes. The textbook view is that these detoxification responses are triggered by chemical recognition or virulence factor receptor proteins (guard proteins) that then couple to signaling pathways for the induction of immunity and detoxification gene responses. Such a toxin recognition surveillance system can be defeated by the evolution of novel bacterial toxins with distinct chemical or protein sequence signatures. But a system that surveils for toxins by their toxicity to, for example, the eukaryotic system targeted, for example, translation of proteins, may be superior for the detection of novel bacterial toxins. The nematode Caenorhabditis elegans surveils a wide range of core cellular pathways for such deficits, and whether it is caused by a toxin, a mutation or RNA interference, detoxification and immunity genes are induced (Melo and Ruvkun, 2012)(Govindan et al., 2015). For example, C. elegans mutations in translation factor genes themselves trigger the same immune and detoxification gene inductions as those induced by bacterial toxins and virulence factors that inhibit $C$. elegans translation (Govindan et al., 2015). Thus, translation in C. elegans is monitored so that decrements in protein synthesis caused by toxins or mutations are detected and coupled to a defense response. The detected translational deficits are coupled via a conserved p38MAPK, bZIP/ZIP-2 transcription factor and the bile acid biosynthetic pathways to activate xenobiotic detoxification and bacterial immune response genes as well as food aversion behaviors (Melo and Ruvkun, 2012)(Govindan et al., 2015). By monitoring decrements in core cellular functions rather than the molecular structure of an unknown toxin, $C$. elegans can detect unanticipated pathogens and toxins. Many of the components of this signaling cascade, for example, the MAP kinase and bile acid biosynthetic pathway, are conserved across animals, suggesting that this system of toxin surveillance and response may not be parochial to $C$. elegans.

Such an animal defensive strategy may drive the evolution of bacterial pathogen countermeasures to thwart these defense responses (Melo and Ruvkun, 2012)(Samuel et al., 
2016). Commensal bacteria may also seek to silence such animal defense responses to establish a benign or symbiotic relationship. Because bacteria synthesize a wide palette of chemical toxins and virulence factors that target the ribosome and associated translation factors (Bérdy, 2005), we reasoned that a screen of diverse bacterial strains could detect counter-surveillance drugs or virulence produced by bacteria. We screened bacterial species by growth of individual bacterial strains with a $C$. elegans strain carrying a mutation in a translation factor gene for bacterial activities that disrupt the induction of xenobiotic detoxification genes. This screen identified an activity of Kocuria rhizophila that suppresses C. elegans surveillance of translation (Govindan et al., 2015). Here we identify by genetic analysis of $K$. rhizophila that $\mathrm{C}_{50}$ carotenoids are the bacterial activity that suppresses the $C$. elegans translational toxin defense response. This $K$. rhizophila activity suppresses the induction of xenobiotic detoxification and food aversion responses caused by deficits in protein synthesis. Another bacterial species that also produces the $\mathrm{C}_{50}$ carotenoid decaprenoxanthin, Corynebacterium glutamicum, also suppressed the induction of xenobiotic detoxification genes in a C. elegans translation factor mutant, and C. glutamicum carotenoid biosynthesis mutants also fail to suppress $C$. elegans detoxification induction. The bacterial inhibition of this detoxification response increases the potency of bacterial toxins that target translation: treatment of wild type $C$. elegans with purified $K$. rhizophila $\mathrm{C}_{50}$ carotenoids caused hypersensitivity to drugs that inhibit translation. Thus, the evolution and lateral transfer between bacterial strains of these carotenoids may have been selected because they increase in the potency of bacterial toxins. Bile acid signaling mediates the signaling between $C$. elegans translational surveillance and induction of drug detoxification genes (Govindan et al., 2015). Addition of mammalian bile acids to the K. rhizophila inhibited pgp-5 response pathway bypassed the carotenoid inhibition of the response, showing that carotenoids act upstream of bile acid production.

\section{Results}

\section{Bacterial carotenoids suppress $C$. elegans surveillance and response to translational deficits}

Like many animal species, C. elegans encodes about 500 xenobiotic detoxification genes ---for example, cytochrome p450, ABC transporter, UDP-glycosyl transferase genes-that are induced by particular toxins or genetic deficits (Melo and Ruvkun, 2012). Particular suites of $C$. elegans detoxification genes are strongly induced by inhibition of ribosomal 
proteins, tRNA synthetases, and other genes implicated in translation (Govindan et al., 2015). The $C$. elegans ABC transporter gene pgp-5 is strongly induced by disruption of ribosomal and other translation factor genes but not by defects in other conserved cellular components such as the mitochondrion or the cytoskeleton (Govindan et al., 2015). A pgp-5::gfp fusion gene is therefore a reporter of $C$. elegans translational dysfunction and not just general poor health or poor growth conditions (Govindan et al., 2015). This ABC transporter protein may eliminate toxins that target the ribosome from the cell, but this has not been established. The translational defect can even be limited to the germline using the C. elegans eft-3(q145) germline-translation defective mutant, which is sterile, but with normal translation in somatic cells. In the eft-3(q145) mutant, $p g p-5 p:: g f p$ is strongly induced in the intestine when the animals are fed the benign E. coli OP50 (Figure S1A; Figure 1A). Feeding K. rhizophila rather than E. coli to $C$. elegans eft-3(q145); pgp-5p::gfp animals disrupts the induction of pgp-5p::gfp (Figure S1A; Figure 1A-B) (Govindan et al., 2015). K. rhizophila is an Actinobacteria, a clade rich in drug biosynthetic pathways. K. rhizophila species are found in soils and rotting fruits associated with natural populations of $C$. elegans in orchards (Samuel et al., 2016). K. rhizophila species are also normal inhabitants of skin and mucous membranes of humans and animals, but can be associated with human infections (Becker et al., 2008) (Moissenet et al., 2012) (Purty et al., 2013).

To establish that $K$. rhizophila can suppress the surveillance of a range of defects in translation, we tested the induction of the pgp-5p::gfp xenobiotic detoxification gene reporter after gene inactivation by RNAi of other genes that encode ribosomal protein subunits or translation factors. For example, growth of wild type C. elegans carrying pgp-5p::gfp on E. coli engineered to produce rpl-1 dsRNA or vrs-2 dsRNA, which inactivate the RPL-1 ribosomal protein or the VRS-2 tRNA synthetase genes, caused induction of pgp-5p::gfp. By contrast, in animals fed on either rpl-1 dsRNA or vrs-2 dsRNA and transferred to $K$. rhizophila, pgp-5p::gfp expression was decreased (Figure S1B-C). The suppression of surveillance pathways by $K$. rhizophila is specific for translational defects because $K$. rhizophila does not suppress the induction of the mitochondrial stress response or endoplasmic reticulum stress response (Govindan et al., 2015). Constant exposure to K. rhizophila is necessary to suppress $C$. elegans translational surveillance: eft-3(q145);pgp$5 p:: g f p$ animals fed on K. rhizophila and transferred after various times to $E$. coli OP50 plates re-expressed pgp-5p::gfp within 12 hours of transfer (Figure S1D). To identify the $K$. rhizophila genetic pathways responsible for the inhibition of pgp-5p::gfp induction by a $C$. 
elegans deficit in translation, we conducted a forward genetic screen after EMS mutagenesis of $K$. rhizophila for bacterial mutant strains that can no longer inhibit pgp-5p::gfp induction in eft-3(q145). 2000 individual K. rhizophila strains that grew normally on bacterial LB plates after EMS mutagenesis were fed to C. elegans eft-3(q145); pgp-5p::gfp animals, one bacterial mutant per well, and screened for $K$. rhizophila mutants that failed to suppress $C$. elegans pgp-5p::gfp induction in a population of 50-100 eft-3(q145); pgp-5p::gfp animals. We identified six K. rhizophila mutant strains that failed to inhibit the induction of pgp-5p::gfp in the eft-3(q145); pgp-5p::gfp strain (Figure S1E-F). All these Kocuria mutant strains had defects in colony pigmentation compared to wild type K. rhizophila (Figure S2A). Wildtype K. rhizophila is yellow, whereas the six mutant colonies were red or white or orange. Using this discoloration phenotype, we visually screened $\sim 500,000$ bacterial colonies after EMS mutagenesis for pigmentation mutants; 71 mutants were identified (Figure S2B). The 71 mutants along with 25 control non-discolored mutants were tested on C. elegans eft-3(q145); pgp-5p::gfp animals and scored for GFP induction. All 71 pigmentation mutants failed to suppress pgp-5p::gfp induction in the $C$. elegans eft-3(q145) mutant, whereas the 25 normally colored K. rhizophila strains suppressed pgp-5p::gfp induction, like wild type $K$. rhizophila (Table S1; Figure S2C; Figure 1A-B).

Genome sequencing of 23 of these pigmentation mutants revealed that each carried a mutation in one of six carotenoid biosynthetic cluster genes (Figure S2D-E). Carotenoids are yellow to red colored pigments, which are produced by a terpenoid biosynthetic pathway (Takarada et al., 2008). The reaction catalyzed by GGPP synthase CrtE, phytoene synthase CrtB and phytoene desaturase Crtl mediate steps in the production of lycopene (Klassen, 2010) (Krubasik et al., 2001a). CrtEb and CrtYe/f cyclases catalyze the biosynthesis of $C_{50}$ carotenoid from lycopene. $\mathrm{C}_{50}$ carotenoids are synthesized more rarely than other carotenoids (Krubasik et al., 2001a). Six missense mutations (e21, e11, e23, e14, e5, and e13) and two nonsense mutations (e15 and e10) were in crtl, which encodes phytoene desaturase (Figure 1C; Figure S2D-E). Crtl catalyzes the conversion of the non-colored phytoene to lycopene, which is red. All these crtl mutants are white colored colonies (Figure S2D; Figure 1C) similar to the Corynebacterium glutamicum $\Delta$ crtl mutant (Heider et al., 2012) (Figure S2F) and thus are predicted to be defective in lycopene synthesis. The six missense mutations are in highly conserved residues suggesting that these are strong loss of function mutations (Figure S3). The e4, e6, and e8 are missense mutations in the crtB gene, which encodes phytoene synthase (Figure 1C; Figure S2D-E). These missense mutations are in 
highly conserved residues, suggesting that these are strong loss of function mutations (Figure S4). The mutants produce white bacterial colonies (Figure S2D; Figure 1C), as was observed in the C. glutamicum $\Delta$ crtB mutant (Heider et al., 2012) (Figure S2F). We obtained four mutations in crtEb; two nonsense mutations (e16 and e17) and two missense mutations (e3 and e19) in highly conserved residues (Figure 1C; Figure S2E; Figure S5). Mutations in crtEb are likely to be defective in the conversion of lycopene to flavuxanthin (Figure 1C). These mutants form pale red colonies (Supplementary Fig. 2d; Fig. 1c) probably because of accumulation of lycopene (but not flavuxanthin), as is seen in the C. glutamicum $\Delta$ crtEb mutant (Heider et al., 2012) (Figure S2F). e17 is an early stop mutation in crtEb which is predicted to produce a truncated protein of 13 amino acids (Figure S5). Mutations in crtYe and crtYf are defective in the last step; the homologs of these genes in C. glutamicum are known to catalyze synthesis of decaprenoxanthin (Krubasik et al., 2001a) (Monnet et al., 2010). Mutations in crtYe and crtYf produce pale red to orange colonies (Figure S2D). A C. glutamicum $\Delta c r t Y$ mutant that deletes both crtYe and crtYf accumulates flavuxanthin and is a pale orange to red color (Heider et al., 2012) (Figure S2F). Even though several bacterial species contain the CrtEb and CrtYe/f genes (Figure S6; See Supplementary methods), the only genetically and biochemically well-characterized $\mathrm{C}_{50}$ carotenoid is decaprenoxanthin from C. glutamicum (Heider et al., 2012). In C. glutamicum, the enzymes CrtEb, CrtYe, and CrtYf convert lycopene to the $\mathrm{C}_{50}$ carotenoid decaprenoxanthin (Krubasik et al., 2001a) (Monnet et al., 2010) (Figure 1C). CrtEb catalyzes the elongation of $\mathrm{C}_{40}$ acyclic lycopene to acyclic $\mathrm{C}_{50}$ carotenoid flavuxanthin (Krubasik et al., 2001a). CrtYe and CrtYf form a $\mathrm{C}_{50}$ cyclase which converts $\mathrm{C}_{50}$ flavuxanthin to decaprenoxanthin (Krubasik et al., 2001a) (Krubasik et al., 2001b). K. rhizophila crtYe and crtYf are 38\% and 34\% identical to C. glutamicum crtYe and crtYf respectively. Therefore, the yellow pigment produced by $K$. rhizophila may belong to the decaprenoxanthin $\mathrm{C}_{50}$-subfamily.

Consistent with the anti-C. elegans translational surveillance activity of $K$. rhizophila decaprenoxanthin, we found that C. glutamicum ATCC13032 which produces a similar $\mathrm{C}_{50}$ pigment also suppressed pgp-5p::gfp induction in eft-3(q145);pgp-5p::gfp (Figure 2A-B). And like the K. rhizophila carotenoid mutants, C. glutamicum ATCC13032 deletion mutants in crtY, crtEb, crtl, and, crtB which disrupt decaprenoxanthin production (Heider et al., 2012) allowed the induction of pgp-5p::gfp in eft-3(q145);pgp-5p::gfp animals (Figure 2A-B). Thus, the C. glutamicum carotenoid mutations also fail to suppress pgp-5p::gfp induction in eft3(q145). 
Arthrobacter arilaitensis, the source of the yellow pigment in Livarot, Maroilles, Munster, Limburger and Tilsit cheeses, also produces decaprenoxanthin (Monnet et al., 2010). Feeding $A$. arilaitensis to eft-3(q145); pgp-5p::gfp animals also suppresses pgp$5 p:: g f p$ induction ((Figure 2C). Thus, C. glutamicum, A. arilaitensis, or K. rhizophila produce a pigmented carotenoid that suppresses translational surveillance, and mutations in $C$. glutamicum or K. rhizophila that disrupt decaprenoxanthin biosynthesis inactivate this suppression of surveillance.

One trivial explanation for the failure of the K. rhizophila carotenoid mutants to suppress pgp-5p::gfp in a translation-defective C. elegans mutant would be that these $K$. rhizophila pigmentation mutants might induce pgp-5p::gfp even in a wildtype C. elegans background. But wild type C. elegans carrying pgp-5p::gfp grown on the K. rhizophila crtEb(e17), crtYe(e22), crtYf(e18), crtB(e6) or crtl(e10) mutants do not induce pgp-5p::gfp (Table S1; Figure S7A). Another possible interpretation was that $K$. rhizophila feeding might induce other stress responses in C. elegans that somehow "distract" the animal from surveillance of translation. We tested the induction of other GFP fusion reporters of stress on wild type and various $K$. rhizophila mutants, and found that $K$. rhizophila wildtype or carotenoid mutants did not induce $h s p-4 p:: g f p$ ((Figure S7B), hsp-6p::gfp (Figure S7C), F35E12.5p::gfp (Figure S7D), or clec-60::gfp (Figure S7E). These stress reporter experiments rule out trivial explanations, such as that Kocuria itself induces the expression of pgp-5::GFP, or that Kocuria induces a distracting stress response so that translation deficits are no longer coupled to pgp-5::GFP induction. These experiments also rule out that the carotenoid pigment of wild type Kocuria absorbs the UV light used to excite GFP fusion genes or the green light emitted by GFP fusion proteins.

\section{Purified carotenoids from Kocuria rhizophila suppress C. elegans translational surveillance}

Carotenoids such as decaprenoxanthin are lipophilic molecules that localize to the cell membrane and can be extracted in non-polar solvents. We extracted wild type K. rhizophila cultures with methanol (Figure S8; Figure 3A-C). TLC analysis of the extract revealed the presence of yellow-orange pigments (Figure 3A, inset). High resolution LC-MS analysis of a methanol extract from $K$. rhizophila showed a complex mixture containing at least six components with similar absorbance spectra that eluted from a reverse phase C18 column (Figure 3A-B; See supplemental methods section). Average absorbance spectra of the 
elution peaks revealed absorption maxima at 414, 438 and $468 \mathrm{~nm}$, which is similar to the published absorption spectra of decaprenoxanthin from Arthrobacter (Giuffrida et al., 2016) (Sutthiwong and Dufossé, 2014). For three of the elution peaks at 19, 16, and 9 min, high resolution mass spectra contained ions that were consistent with decaprenoxanthin and its mono- and di-glucosides, respectively, with mass accuracy within 2 ppm (Figure 3C). Three other peaks with similar absorbance spectra were not assignable by mass.

To analyze the carotenoid production in the $K$. rhizophila mutants, we extracted

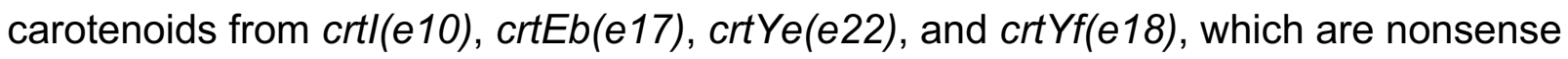
mutant alleles and $\operatorname{crtB}(e 6)$, a missense mutant. Spectrophotometric analysis of methanol extracts from wildtype $K$. rhizophila showed absorption maxima of $415-425 \mathrm{~nm}$, whereas the crtEb(e17) extract showed absorption maxima at 445-455nm (Figure S9). K. rhizophila crtEb(e17) and crtYe(e22) mutant methanol extract show similar absorption spectra while the extract from K. rhizophila crtl(e10) and crtb(e6) showed no absorption at all. The methanol extracts from $\operatorname{cr} Y f(e 18)$ showed two separate absorption peaks one at $\sim 400 \mathrm{~nm}$ and another minor one at $\sim 500 \mathrm{~nm}$. To determine the identities of these absorption peaks in the mutants, we conducted LC-MS analysis of carotenoid extracts from crtYe(e22) and crtEb(e17) (Figure S10A-C). Mutations in crtEb are predicted to block the synthesis of carotenoids at the flavuxanthin biosynthesis step and thus are likely to accumulate lycopene (Figure S10C). Consistent with this prediction, MS spectral analysis revealed that crtEb(e17) mutants accumulate lycopene. Mutations in crtYe are predicted to block the synthesis of carotenoids at the decaprenoxanthin biosynthesis step and thus likely to accumulate flavuxanthin (Figure $\mathrm{S10C})$. However, we found that crtYe(e22) mutants accumulate lycopene as well as other unidentified peaks.

We tested whether the crude methanol extracts from wildtype $K$. rhizophila, containing pigmented carotenoids, could suppress GFP induction in the germline-translation defective $C$. elegans eft-3(q145);pgp-5p::gfp animals. Animals fed on E. coli with the K. rhizophila carotenoid extract exhibited significantly reduced pgp-5p::gfp expression compared to eft3(q145);pgp-5p::gfp animals fed on E. coli with control methanol extract (Figure 3D). The wild type $K$. rhizophila carotenoid methanol extract could also rescue the failure to suppress C. elegans surveillance by K. rhizophila carotenoid biosynthetic mutants: when eft3(q145);pgp-5p::gfp animals were fed on K. rhizophila crtEb(e17), crtB(e6) or crtl(e10) mutants supplemented with wildtype $K$. rhizophila methanol extract, GFP was not induced 
while in the animals fed on control extract, the GFP expression was induced by the $C$. elegans eft-3 mutation (Figure 4A).

Because $\mathrm{C}_{50}$ carotenoids have multiple conjugated double bonds, they are likely to be antioxidants (Edge et al., 1997). Several carotenoids including $\mathrm{C}_{30}, \mathrm{C}_{40}$, or $\mathrm{C}_{50}$ carotenoids are antioxidants. However, it is unlikely that the ROS-quenching property of carotenoids is responsible for the suppression of pgp-5p::gfp induction. First, multiple antioxidants do not suppress the induction of pgp-5p::gfp in a $C$. elegans translation defective mutant: eft3(q145);pgp-5p::gfp animals grown on E. coli OP50 with $\mathrm{N}$-acetyl cysteine, ascorbic acid, Trolox, or resveratrol induce pgp-5p::gfp normally (Figure S11A). Second, pgp-5p::gfp is not induced by oxidative stress (Govindan et al., 2015).

Consistent with the genetic analysis of $K$. rhizophila and C. glutamicum highlighting the production of $\mathrm{C}_{50}$ decaprenoxanthin as key to suppression of $C$. elegans immune responses, we found that other commercially available 40 carbon carotenoids in the pathway to decaprenoxanthin $\mathrm{C}_{50}$ biosynthesis cannot suppress the induction of pgp-5p::gfp in a $C$. elegans translation defective mutant. For example, treating eft-3(q145);pgp-5p::gfp animals grown on $E$. coli OP50 with either $\mathrm{C}_{40}$ beta-carotene or $\mathrm{C}_{40}$ astaxanthin did not suppress pgp-5p::gfp induction (Figure $\mathrm{S} 11 \mathrm{~B}$ ). $\mathrm{C}_{40}$ zeaxanthin, $\mathrm{C}_{40}$ neurosporene, $\mathrm{C}_{40}$ violaxanthin, $\mathrm{C}_{40}$ delta-carotene, or $\mathrm{C}_{40}$ alpha-carotene also did not suppress pgp-5p::gfp induction (Figure $\mathrm{S} 11 \mathrm{C})$. These $\mathrm{C}_{40}$ carotenoids cannot suppress the response to translational deficits like the $\mathrm{C}_{50}$ carotenoids produced by K. rhizophila and C. glutamicum.

\section{Suppression of C. elegans translational surveillance by Kocuria rhizophila carotenoids enhances the toxicity of ribosomal toxins}

K. rhizophila also suppresses the C. elegans detoxification response to translation inhibiting drugs. Hygromycin is a bacterially produced antibiotic (from Streptomyces hygroscopicus) that inhibits translation and induces xenobiotic detoxification in C. elegans. While $10 \mu \mathrm{g} / \mathrm{ml}$ of hygromycin induces pgp-5p::gfp expression in wild type animals grown on E. coli OP50, wild type animals grown on $K$. rhizophila and $10 \mu \mathrm{g} / \mathrm{ml}$ of hygromycin fail to induce pgp-5p::gfp (Figure S7A; Figure S11D). However, at high concentrations of hygromycin, pgp-5p::gfp is induced both in animals fed on E. coli OP50 or K. rhizophila (Figure S11D). By contrast, wild type C. elegans carrying pgp-5p::gfp grown on the $K$. rhizophila carotenoid biosynthesis mutants crtl(e10) or crtEb(e17) induced GFP at the low concentration of $10 \mu \mathrm{g} / \mathrm{ml}$ of hygromycin unlike animals grown on wild type $K$. rhizophila that 
produces the carotenoid (Figure S7A). Similar results were obtained with emetine which blocks protein synthesis by binding to the 40 S subunit of the ribosome; wild type $K$. rhizophila suppressed the pgp-5p::gfp induction by a low dose of emetine but the carotenoid mutant $K$. rhizophila now allowed induction of pgp-5p::gfp by a low dose of emetine (Figure S12A-B).

We tested whether the suppression of drug detoxification responses by $K$. rhizophila carotenoids increase $C$. elegans sensitivity to translational inhibitors. While wildtype animals grown on $10 \mu \mathrm{g} / \mathrm{ml}$ hygromycin when fed E. coli OP50 are not growth inhibited, animals grown on $10 \mu \mathrm{g} / \mathrm{ml}$ hygromycin plus K. rhizophila carotenoid extract when fed E. coli OP50 are strongly growth inhibited (Figure 4B; Figure S12C). Carotenoids without hygromycin are not toxic to the worms (Figure 4B; Figure S12C). Similar results were obtained with emetine: animals treated with K. rhizophila extracts were hypersensitive to emetine compared to animals fed on control extract (Figure 4C). The xenobiotic hypersensitivity phenotype is specific to translation defects because $K$. rhizophila carotenoid extract does not alter the sensitivity of animals to antimycin, a mitochondrial poison (Figure S12E).

pgp-5p::gfp is also induced in response to genotoxic stress: cisplatin, which interferes with DNA replication, induces pgp-5p::gfp. While $1 \mathrm{mM}$ cisplatin induces pgp-5p::gfp expression in wild type C. elegans grown on E. coli OP50, wild type C. elegans grown on $K$. rhizophila and $1 \mathrm{mM}$ cisplatin fail to induce pgp-5p::gfp (Figure S12D). By contrast, pgp5p::gfp animals fed K. rhizophila crtl(e10), crtEb(e17), or crtYe(e22) carotenoid mutant bacteria induce pgp-5p::gfp in response to $1 \mathrm{mM}$ cisplatin normally (Figure S12D). And the genotoxic response is salient for survival of cisplatin: animals treated with $K$. rhizophila extracts were hypersensitive to cisplatin compared to animals fed on control extract (Figure 4D).

C. elegans food aversion behaviors are induced when animals are exposed to xenobiotics or essential gene inactivations (Melo and Ruvkun, 2012). Exposing animals to hygromycin or cisplatin induces strong food aversion, and this food aversion is modulated by K. rhizophila carotenoid extract (Figure $4 \mathrm{E}-\mathrm{F}$ ). While $\sim 40 \%$ of animals exposed to $25 \mu \mathrm{g} / \mathrm{ml}$ hygromycin display food aversion behavior, only $\sim 15 \%$ of animals exposed to $25 \mu \mathrm{g} / \mathrm{ml}$ hygromycin and K. rhizophila carotenoid extract show aversion (Figure 4E). Similar results were obtained with cisplatin: $\sim 50 \%$ of animals exposed to $1 \mathrm{mM}$ cisplatin display aversion behavior while $<20 \%$ of animals exposed to $1 \mathrm{mM}$ cisplatin and $K$. rhizophila carotenoid extract display food aversion (Figure 4F). 


\section{C. elegans pathway analysis of $K$. rhizophila inhibition of translational surveillance}

How do K. rhizophila carotenoids inhibit the induction of xenobiotic detoxification response pathways? To address this question, we conducted genetic epistasis analysis with C. elegans mutations that disrupt or activate the signal transduction pathway for translational surveillance at various steps (Govindan et al., 2015). The zip-2/bZIP transcription factor is required for the induction of pgp-5p::gfp expression in response to translation inhibition (Govindan et al., 2015). Constitutive expression of ZIP-2::mCherry from an intestine-specific promoter fusion is sufficient to induce pgp-5p::gfp expression in wildtype $C$. elegans without translation inhibition (Fig. 5a,b). pgp-5p::gfp induction in this ZIP-2::mCherry constitutive expression strain was similar in animals fed on E. coli OP50 or wild type K. rhizophila (Figure A-B), suggesting that the $K$. rhizophila carotenoids disrupt a surveillance pathway component upstream of the production of the ZIP-2 bZIP transcription factor.

The induction of $C$. elegans xenobiotic detoxification genes by translation inhibition depends on multiple steps in the bile acid biosynthetic pathway from cholesterol (Govindan et al., 2015). C. elegans with defects in bile acid biosynthesis fail to activate pgp-5p::gfp in response to inhibition of translation. Although the $C$. elegans bile acid biosynthetic pathway is necessary for pgp-5p::gfp in response to inhibition of translation, bile acids are not sufficient to induce pgp-5p::gfp in wildtype animals (Govindan et al., 2015). But addition of mammalian bile acids to these $C$. elegans bile acid pathway mutants substitutes for the missing endogenous bile acids to reanimate this translational defect signal (Govindan et al., 2015). While K. rhizophila feeding inhibits the induction of pgp-5p::gfp in eft-3(q145) animals, addition of exogenous mammalian bile acids reactivates GFP expression when feeding on wild type K. rhizophila (Figure 5C-D). Thus, K. rhizophila carotenoids act either upstream or at the bile acid signaling step of this $C$. elegans translational surveillance and response pathway.

\section{Discussion}

We have found that multiple actinobacterial species produce $\mathrm{C}_{50}$ carotenoid pigments that are potent inhibitors of the animal surveillance pathways for bacterial toxins and virulence factors which target translation. We genetically dissected how the actinobacteria Kocuria rhizophila inhibits the surveillance and detoxification response of $C$. elegans to translational deficits. Our bacterial genetic analysis identified the carotenoid biosynthetic pathway as the key bacterial countermeasure. We isolated mutants in the K. rhizophila $\mathrm{C}_{50}$ 
carotenoid biosynthetic pathway that failed to inhibit this $C$. elegans xenobiotic detoxification response (Figure 1A-C). Hydrophobic and pigmented extracts of $K$. rhizophila enriched for $\mathrm{C}_{50}$ carotenoid suppressed $C$. elegans xenobiotic detoxification response and restored the ability of $K$. rhizophila carotenoid mutants to suppress the $C$. elegans response to defects in translation (Figure 2B-C). The $K$. rhizophila extracts also suppress $C$. elegans detoxification of toxins that target translation or DNA damage, rendering the animal far more sensitive to these toxins (Figure 4B-D, Figure S12C). Thus, carotenoid biosynthesis increases the potency of other bacterial toxins or virulence factors that might target translation or DNA.

A distantly related bacterium, Corynebacterium glutamicum, also suppresses this $C$. elegans xenobiotic detoxification response. Mutations that disable steps in the synthesis of this carotenoid no longer suppress $C$. elegans translational deficit responses--showing that $\mathrm{C}_{50}$ carotenoid regulation of the $C$. elegans surveillance is not limited to one particular strain of bacteria. $\mathrm{C}_{50}$ carotenoids suppress the induction of xenobiotic detoxification by inhibiting $C$. elegans bile acid signaling, which transduce translational deficits to defense responses (Govindan et al., 2015) (Figure 5C-D). The lipid solubility of the bacterial carotenoids and their abundance may be a key feature of their anti-bile acid signaling function in $C$. elegans surveillance of translational and DNA damage response.

The evolutionary rationale for the disabling of eukaryotic toxin response pathways by bacteria is that they could enhance the toxicity of ribosomal toxins. But neither wild type $K$. rhizophila nor the $\mathrm{C}_{50}$ biosynthesis mutants show evidence of translational inhibitor production (for example induction of C. elegans pgp-5::gfp). It is possible that K. rhizophila generates other toxins that cause deficits not related to translation, which are rendered less defended by the associated $\mathrm{C}_{50}$ production. Alternatively, $K$. rhizophila may associate with other bacteria that produce toxins or virulence factors that target the animal translational apparatus or cause DNA damage.

Carotenoids have been most studied in photosynthetic bacteria and plants, where they are auxillary light absorbing components in photosynthesis (Edge et al., 1997). Because carotenoids are antioxidants and light absorbing in photosynthetic bacteria and plants, the literature on carotenoids is highly focused on photosynthesis, light absorption, and free radical reactivity. In photosynthetic chlorophyll clusters, the carotenoids absorb and transduce light energy to photosynthetic electron transport systems to generate a $\mathrm{pH}$ gradient. In photosynthetic chlorophyll clusters, the carotenoids absorb and efficiently transduce light energy to photosynthetic electron transport systems. What is common 
between the K. rhizophila and C. glutamicum and photosynthetic bacteria is the strong absorbance in the visible light wavelengths and the lipid solubility of these pigments. These bacteria are highly colored because the carotenoids are both highly abundant and because they have conjugated double bond systems. Carotenoids are also antioxidants in photosynthesis. However, a simple antioxidant activity of carotenoids does not explain the suppression of the animal drug detoxification response because other antioxidants could not suppress $C$. elegans surveillance.

How do the $\mathrm{C}_{50}$ carotenoids of $K$. rhizophila and C. glutamicum inhibit animal surveillance of their translation competence? The simplest model is that $\mathrm{C}_{50}$ carotenoids induce a change in membrane fluidity to change cell signaling via bile acids. We found that the K. rhizophila $\mathrm{C}_{50}$ carotenoids suppress the induction of the $C$. elegans xenobiotic detoxification response by inhibiting a bile acid biosynthetic pathway. Although bile acids were traditionally thought to be emulsifiers of fat, thus aiding their enzymatic metabolism, bile acids are also signaling molecules in metabolism and immune pathways (Fiorucci et al., 2018). The lipid solubility of carotenoids may be a key feature of their anti-bile acid signaling function in $C$. elegans surveillance of translational and DNA damage response.

Emulsification of lipids by bile acids may be relevant to the antagonistic relationship we show for bile acid signaling in $C$. elegans and the bacterial carotenoid inhibitors of that signaling pathway.

It is possible that the pigmentation of the $\mathrm{C}_{50}$ carotenoids, the particular wavelengths of light absorbance, are key to their anti-surveillance function. For example, our genetic analysis showed that the carotenoids of $K$. rhizophila or $C$. glutamicum do not function at lengths less than $\mathrm{C}_{50}$, suggesting that simple hydrophobicity of $\mathrm{C}_{40}$ or smaller carotenoids is not sufficient to mediate K. rhizophila or C. glutamicum inhibition of C. elegans translational surveillance. The specific color absorbance of the $\mathrm{C}_{50}$ carotenoids (their red color) may mediate their $C$. elegans anti-surveillance activity. For example, C. elegans with a translational deficit may produce blue light (Ohmiya and Hirano, 1996), and the absorption of such light by $\mathrm{C}_{50}$ carotenoids could suppress a light-responsive $C$. elegans surveillance pathway. Such a system might function in the dark of the soil or rotting fruit or night, but might be overwhelmed by sunlight.

Translation surveillance not only induces detoxification but also food aversion behaviors in $C$. elegans. Aversion to food associated with a toxin is an appropriate animal response since many toxins originate from bacterial pathogens that cause the rotting of food. 
Aversion to toxins is a common animal response since many toxins originate from a pathogen, and this response is likely to be an animal program derived from this evolutionary history. K. rhizophila C50 carotenoids suppressed the food aversion induced by emetogenic toxins, emetine or cisplatin. Cisplatin, which is used to block DNA replication in cancer patients, also has high emetogenic potential. Emetine, which is an antibiotic that targets eukaryotic protein synthesis, is also highly emetogenic as the name itself implies. These two drugs not only induce $C$. elegans xenobiotic detoxification but also strong food aversion behavior, both of which are strongly suppressed by bacterial $\mathrm{C}_{50}$ carotenoids. Chemotherapy-induced nausea and vomiting (CINV) in humans may be related to these xenobiotic aversion programs. The $K$. rhizophila $\mathrm{C}_{50}$ carotenoid decaprenoxanthin might for example suppress CINV, perhaps as an adjuvant in chemotherapy. We have hypothesized previously that autoimmune disorders may be triggered by reduction of function mutations in core cellular pathways such as translation that activate these surveillance and detoxification pathways (Govindan et al., 2015). Because the $\mathrm{C}_{50}$ carotenoid decaprenoxanthin robustly suppresses $C$. elegans translational surveillance, it may suppress the inappropriate autoimmune responses to such mutations in mammals. More broadly, bacterial gene activities that suppress animal toxin surveillance pathways could neutralize the toxicity that often plagues drug development.

\section{Acknowledgments}

We thank members of the G.R. laboratory for helpful discussions. The work was supported by NIH Grant AG043184 (to G.R.). Some strains were provided by the CGC, which is funded by NIH Office of Research Infrastructure Programs (P40 OD010440).

\section{References:}

Becker, K., Rutsch, F., Uekötter, A., Kipp, F., König, J., Marquardt, T., Peters, G., and von Eiff, C. (2008). Kocuria rhizophila adds to the emerging spectrum of micrococcal species involved in human infections. Journal of Clinical Microbiology 46, 3537-3539.

Bérdy, J. (2005). Bioactive microbial metabolites. The Journal of Antibiotics 58, 1-26.

Bolz, D.D., Tenor, J.L., and Aballay, A. (2010). A conserved PMK-1/p38 MAPK is required in caenorhabditis elegans tissue-specific immune response to Yersinia pestis infection. Journal of Biological Chemistry 285, 10832-10840. 
Calfon, M., Zeng, H., Urano, F., Till, J.H., Hubbard, S.R., Harding, H.P., Clark, S.G., and Ron, D. (2002). IRE1 couples endoplasmic reticulum load to secretory capacity by processing the XBP-1 mRNA. Nature 415, 92-96.

Edge, R., McGarvey, D.J., and Truscott, T.G. (1997). The carotenoids as anti-oxidants-a review. Journal of Photochemistry and Photobiology. B, Biology 41, 189-200.

Fiorucci, S., Biagioli, M., Zampella, A., and Distrutti, E. (2018). Bile Acids Activated Receptors Regulate Innate Immunity. Frontiers in Immunology 9, 1853.

Giuffrida, D., Sutthiwong, N., Dugo, P., Donato, P., Cacciola, F., Girard-Valenciennes, E., Le Mao, Y., Monnet, C., Fouillaud, M., Caro, Y., et al. (2016). Characterisation of the C50 carotenoids produced by strains of the cheese-ripening bacterium Arthrobacter arilaitensis. International Dairy Journal 55, 10-16.

Govindan, J.A., Jayamani, E., Zhang, X., Breen, P., Larkins-Ford, J., Mylonakis, E., and Ruvkun, G. (2015). Lipid signalling couples translational surveillance to systemic detoxification in Caenorhabditis elegans. Nature Cell Biology 17, 1294-1303.

Heider, S.A.E., Peters-Wendisch, P., and Wendisch, V.F. (2012). Carotenoid biosynthesis and overproduction in Corynebacterium glutamicum. BMC Microbiology 12, 198.

Klassen, J.L. (2010). Phylogenetic and evolutionary patterns in microbial carotenoid biosynthesis are revealed by comparative genomics. PloS One 5, e11257.

Krubasik, P., Takaichi, S., Maoka, T., Kobayashi, M., Masamoto, K., and Sandmann, G. (2001a). Detailed biosynthetic pathway to decaprenoxanthin diglucoside in Corynebacterium glutamicum and identification of novel intermediates. Archives of Microbiology 176, 217-223.

Krubasik, P., Kobayashi, M., and Sandmann, G. (2001b). Expression and functional analysis of a gene cluster involved in the synthesis of decaprenoxanthin reveals the mechanisms for C50 carotenoid formation. European Journal of Biochemistry 268, 3702-3708.

Melo, J.A., and Ruvkun, G. (2012). Inactivation of conserved C. elegans genes engages pathogen- and xenobiotic-associated defenses. Cell 149, 452-466.

Moissenet, D., Becker, K., Mérens, A., Ferroni, A., Dubern, B., and Vu-Thien, H. (2012). Persistent bloodstream infection with Kocuria rhizophila related to a damaged central catheter. Journal of Clinical Microbiology 50, 1495-1498.

Monnet, C., Loux, V., Gibrat, J.-F., Spinnler, E., Barbe, V., Vacherie, B., Gavory, F., Gourbeyre, E., Siguier, P., Chandler, M., et al. (2010). The arthrobacter arilaitensis Re117 genome sequence reveals its genetic adaptation to the surface of cheese. PloS One 5, e15489.

Ohmiya, Y., and Hirano, T. (1996). Shining the light: the mechanism of the bioluminescence reaction of calcium-binding photoproteins. Chemistry \& Biology 3, 337-347.

O’Rourke, D., Baban, D., Demidova, M., Mott, R., and Hodgkin, J. (2006). Genomic clusters, putative pathogen recognition molecules, and antimicrobial genes are induced by infection of $\mathrm{C}$. elegans with M. nematophilum. Genome Research 16, 1005-1016. 
Purty, S., Saranathan, R., and Prashanth, K. (2013). The expanding spectrum of human infections caused by Kocuria species: a case report and literature review. \ldots Microbes \& Infections.

Samuel, B.S., Rowedder, H., Braendle, C., Félix, M.-A., and Ruvkun, G. (2016). Caenorhabditis elegans responses to bacteria from its natural habitats. PNAS 113, E3941-E3949.

Sutthiwong, N., and Dufossé, L. (2014). Production of carotenoids by Arthrobacter arilaitensis strains isolated from smear-ripened cheeses. FEMS Microbiology Letters 360, 174-181.

Takarada, H., Sekine, M., Kosugi, H., Matsuo, Y., Fujisawa, T., Omata, S., Kishi, E., Shimizu, A., Tsukatani, N., Tanikawa, S., et al. (2008). Complete genome sequence of the soil actinomycete Kocuria rhizophila. Journal of Bacteriology 190, 4139-4146.

Troemel, E.R., Chu, S.W., Reinke, V., Lee, S.S., Ausubel, F.M., and Kim, D.H. (2006). p38 MAPK regulates expression of immune response genes and contributes to longevity in C. elegans. PLoS Genetics 2, e183.

Yoneda, T., Benedetti, C., Urano, F., Clark, S.G., Harding, H.P., and Ron, D. (2004). Compartmentspecific perturbation of protein handling activates genes encoding mitochondrial chaperones. Journal of Cell Science 117, 4055-4066. 


\section{Figure 1: K.rhizophila carotenoid biosynthetic mutants fail to suppress $C$. elegans surveillance of translational deficits}

A) pgp-5p::gfp induction was significantly reduced in eft-3(q145); pgp-5p::gfp animals cultivated on $K$. rhizophila wildtype while mutants in K. rhizophila crtEb(e17), K. rhizophila crtl(e10), and K. rhizophila crtYe(e2) did not suppress the GFP induction.

B) K. rhizophila feeding reduced pgp-5p::gfp induction in eft-3(q145); pgp-5p::gfp animals significantly, while in the K. rhizophila mutants, pgp-5p::gfp expression in this strain was not affected. Unpaired t-test, ${ }^{* *} P<0.01$. Mean $\pm s . d$ is shown. The number of animals analyzed per condition is shown above each bar. ns, was not significant compared to eft-3(q145); pgp-5p::gfp fed on E. coli OP50

C) The Kocuria rhizophila $\mathrm{C}_{50}$ carotenoid biosynthetic pathway. The genome of $K$. rhizophila contains an operon that encodes predicted carotenoid (crt) biosynthetic genes: crtE (KRH_20850; encoding GGPP synthase), crtB (KRH_20840; encoding phytoene synthase), crtl (KRH_20830; encoding phytoene desaturase), crtEb (KRH_20800; encoding lycopene elongase), crtYe (KRH_20820; encoding $\mathrm{C}_{50}$ carotenoid epsilon cyclase subunit) and crtYf (KRH_20810; encoding $\mathrm{C}_{50}$ carotenoid epsilon cyclase subunit). The crtYe and crtYf subunits form a heterodimeric cyclase complex that catalyzes generation of $\mathrm{C}_{50}$ carotenoid. Colonies of wildtype $\mathrm{K}$. rhizophila and mutants are shown. While wildtype $K$. rhizophila colonies were yellow, crtB and crtl mutant colonies were colorless. crtYe, crtYf, and crtEb mutant colonies were various shades of red.

\section{Figure 2: Bacterial C50 carotenoids suppress $C$. elegans surveillance of translational deficits}

A) pgp-5p::gfp induction was significantly reduced in eft-3(q145); pgp-5p::gfp animals grown on C. glutamicum wildtype while mutants in C. glutamicum $\Delta c r t E b, \Delta c r t I, \Delta c r t Y$, and $\triangle c r t B$ did not suppress the GFP induction.

B) Quantification of $p g p-5 p:: g f p$ expression in eft-3(q145); pgp-5p::gfp animals grown on C. glutamicum wildtype, $\Delta c r t E b, \Delta c r t l, \Delta c r t Y$, and $\Delta c r t B$ mutants. Unpaired t-test, ${ }^{* * * * P} P<0.0001$. Mean $\pm s . d$ is shown. The number of animals analyzed per condition is 
shown above each bar. ns, was not significant compared to eft-3(q145); pgp-5p::gfp fed on E. coli OP50.

C) pgp-5p::gfp induction was significantly reduced in eft-3(q145); pgp-5p::gfp animals grown on wildtype $A$. arilaitensis.

\section{Figure 3: LC-MS analysis of carotenoid extracts from $K$. rhizophila}

A) Chromatogram of $440 \mathrm{~nm}$ absorbance after reverse phase LC separation of clarified extracts on a C18 column. Six major elution peaks are indicated, Inset: TLC of $K$. rhizophila extract showing yellow-orange pigments.

B) Average absorbance spectra for each indicated elution peak. Spectra are normalized and baseline shifted for clarity. All six elution peaks share similar absorbance spectra, but three peaks (grey lettering) were not conclusively assigned by mass.

C) Background-subtracted averaged MS spectra of three assigned elution peaks at approximately 9 (green), 16 (orange), and 19 min (dark grey) with observed neutral monoisotopic mass (obs.), corresponding exact calculated mass (calc.), and mass error for each indicated chemical formula. Each spectrum shows the calculated isotopic distribution for the identified adduct ions (red bars).

D) $750 \mu \mathrm{g} / \mathrm{ml}$ of $K$. rhizophila extract inhibited pgp-5p::gfp induction in eft-3(q145); pgp5p::gfp animals.

\section{Figure 4: Purified carotenoids from Kocuria rhizophila suppress C. elegans translational surveillance}

A) Quantification of pgp-5p::gfp expression in eft-3(q145); pgp-5p::gfp animals fed on $K$. rhizophila wildtype, K. rhizophila crtEb(e17), K. rhizophila crtl(e10), and K. rhizophila crtEb(e6) supplemented with either control extract or an extract from wild type $K$. rhizophila.

B) Animals treated with K. rhizophila carotenoid extract were hypersensitive to the translational inhibitor hygromycin. Unpaired t-test, ${ }^{* * *} \mathrm{P}<0.0001$ compared to the wildtype worms fed on E. coli OP50 containing solvent extract and hygromycin. Mean 
\pm s.d is shown. Data were collected from three independent trials of at least 20 animals for each condition. ns, was not significant compared to wildtype grown on $E$. coli OP50 containing solvent extract with no hygromycin

C) Animals treated with K. rhizophila carotenoid extract were hypersensitive to emetine.

D) Animals treated with K. rhizophila carotenoid extract were hypersensitive to cisplatin.

E) Animals treated with K. rhizophila carotenoid extract failed to show aversive behaviors on hygromycin compared animals treated with control solvent and hygromycin.

F) Animals treated with K. rhizophila carotenoid extract failed to avoid cisplatin-compared animals treated with control solvent and cisplatin. Unpaired t-test, ${ }^{* * * *} \mathrm{P}<0.01$, ${ }^{* *} P<0.0001$. Mean \pm s.d is shown. ns, not significant compared to

\section{Figure 5: C. elegans pathway analysis of $K$. rhizophila inhibition of translational surveillance}

A) pgp-5p::gfp was constitutively induced in animals fed on E. coli OP50 or K. rhizophila expressing ZIP-2::mCherry in the intestine under the control of the vha-6 promoter.

B) Quantification of pgp-5p::gfp activation in animals expressing ZIP-2::mCherry in the intestine under the control of vha-6 promoter. Compared to the pgp-5p::gfp animals fed on E. coli OP50, animals expressing ZIP-2::mCherry in the intestine under the control of vha-6 promoter had increased pgp-5p::gfp levels when fed on either $E$. coli OP50 or K. rhizophila. Mean \pm s.d is shown. The black dots on each bar indicates each animal analyzed per condition.

C) Supplementation of bile acids suppressed the K. rhizophila pgp-5p::gfp activation defect in eft-3(q145); pgp-5p::gfp animals.

D) Quantification of the suppression of the K. rhizophila pgp-5p::gfp activation defect in eft-3(q145); pgp-5p::gfp animals by bile acids shown in Figure 4B. Unpaired t-test, ${ }^{* * *} P<0.0001$. Mean \pm s.d is shown. The number of animals analyzed per condition is 
shown above each bar. ns, was not significant compared to eft-3(q145); pgp-5p::gfp fed on E. coli OP50.

\section{Supplementary Figure 1: K.rhizophila carotenoid biosynthetic mutants fail to suppress pgp-5::gfp induction}

A) K. rhizophila feeding inhibited pgp-5p::gfp induction in eft-3(q145); pgp-5p::gfp animals within 12 hours of feeding.

B) While pgp-5p::gfp animals fed on vrs-2 dsRNA and transferred to E. coli showed induction of gfp, in animals transferred to K. rhizophila plates, pgp-5p::gfp expression was reduced.

C) While pgp-5p::gfp animals fed on rpl-1 dsRNA and transferred to E. coli showed induction of gfp, in animals transferred to K. rhizophila plates, pgp-5p::gfp expression was reduced.

D) K. rhizophila feeding suppressed pgp-5p::gfp induction in eft-3(q145); pgp-5p::gfp animals was reversible.

E) K. rhizophila feeding reduced pgp-5p::gfp induction in eft-3(q145); pgp-5p::gfp animals significantly, while in the K. rhizophila mutants, pgp-5p::gfp expression is not affected.

F) K. rhizophila feeding inhibited the induction of pgp-5p::gfp in eft-3(q145); pgp-5p::gfp animals while in the K. rhizophila mutants, pgp-5p::gfp expression was not affected.

\section{Supplementary Figure 2: K. rhizophila carotenoid pathway is required for inhibition of} pgp-5p::gfp induction in eft-3(q145); pgp-5p::gfp animals

A) Colony color of $K$. rhizophila wildtype was different from the colony color of the six mutants.

B) Colony color of the K. rhizophila mutants observed from the EMS screen.

C) K. rhizophila pigmentation mutants failed to suppress the induction of $p g p-5 p:: g f p$ in eft-3(q145); pgp-5p::gfp animals.

D) The pigmentation defect in 23 genome sequenced mutants.

E) Predicted K. rhizophila carotenoid (crt) biosynthetic genes: crtE (KRH_20850; encoding GGPP synthase), crtB (KRH_20840; encoding phytoene synthase), crtI 
(KRH_20830; encoding phytoene desaturase), crtEb (KRH_20800; encoding lycopene elongase), crtYe (KRH_20820; encoding $\mathrm{C}_{50}$ carotenoid epsilon cyclase subunit) and crtYf (KRH_20810; encoding $\mathrm{C}_{50}$ carotenoid epsilon cyclase subunit). The crtYe and crtYf subunits form a heterodimeric cyclase complex that catalyzes generation of $\mathrm{C}_{50}$ carotenoid are shown with the genomic locus coordinates. The genomic location of the mutation with the type of change in amino acid is shown for each mutant.

F) Pigmentation defects observed from C. glutamicum wildtype, $\Delta c r t E b, \Delta c r t$, $\Delta c r t Y$, and $\Delta$ crtB mutants.

\section{Supplementary Figure 3: Alignment of Crtl protein in different species}

Alignment of Crtl protein in different species. The amino acid conservation across different species and the mutations isolated are shown. Protein sequences were obtained from NCBI and the sequences were aligned using CLUSTAL Omega and BOXSHADE server.

\section{Supplementary Figure 4: Alignment of CrtB protein in different species}

Alignment of CrtB protein in different species. The amino acid conservation across different species and the mutations isolated are shown. Protein sequences were obtained from NCBI and the sequences were aligned using CLUSTAL Omega and BOXSHADE server.

\section{Supplementary Figure 5: Alignment of CrtEb protein in different species}

Alignment of CrtEb protein in different species. The amino acid conservation across different species and the mutations isolated are shown. Protein sequences were obtained from NCBI and the sequences were aligned using CLUSTAL Omega and BOXSHADE server.

\section{Supplementary Figure 6: Operon structure of bacteria that contain putative gene cluster that might produce decaprenoxanthin} Genomic sequences were obtained from NCBI and analyzed for the presence of operons manually.

\section{Supplementary Figure 7: Testing whether $K$. rhizophila wildtype or carotenoid mutants regulate other stress pathways}


A) pgp-5p::gfp induction in response to $10 \mu \mathrm{g} / \mathrm{ml}$ of hygromycin was significantly reduced in animals grown on $K$. rhizophila wildtype while mutants in $K$. rhizophila crtEb(e17), or K. rhizophila crtl(e10) did not suppress the GFP induction by hygromycin.

B) K. rhizophila did not induce $h s p-4 p:: g f p$. $h s p-4 p:: g f p$ is a reporter of mitochondrial unfolded protein response (UPR ${ }^{\text {mito }}$ )(Calfon et al., 2002).

C) K. rhizophila wildtype as well as $\operatorname{crtEb}(e 17)$ or $\operatorname{crtl}(e 10)$ mutants did not induce $h s p$ $6 p:: g f p$. hsp-6p::gfp is a reporter of mitochondrial unfolded protein response $\left(\right.$ UPR $^{\text {mito }}$ )(Yoneda et al., 2004).

D) K. rhizophila wildtype as well as crtEb(e17) or crtl(e10) mutants did not induce F35E12.5p::gfp. F35E12.5p::GFP is a CUB domain protein induced by Y. pestis, $M$. nematophilum and $P$. aeruginosa (Bolz et al., 2010) (O'Rourke et al., 2006) (Troemel et al., 2006).

E) K. rhizophila wildtype as well as crtEb(e17), crtl(e10), crtYf(e2) or crtYe(e22) mutants induced clec-60p::gfp. clec-60 is a C-type lectin/CUB domain protein induced by the gram positive pathogens, S. aureus and M. nematophilum (O'Rourke et al., 2006). clec-60::GFP is induced by gram-positive bacteria. Because K. rhizophila is also a gram-positive bacterium, the induction of clec-60 may be an immune response to a pathogen.

\section{Supplementary Figure 8: Biochemical isolation of decaprenoxanthin containing extract from K. rhizophila}

K. rhizophila cultures grown in LB solution was washed with equal volume of water after centrifugation at 4000RPM for $15 \mathrm{~min}$. After centrifugation to remove water, equal volume of acetone was added and centrifuged again at 4000RPM for $15 \mathrm{~min}$. After removal of acetone, the bacterial pellets were extracted with methanol at $65^{\circ} \mathrm{C}$ in water bath after wrapping the samples with aluminum foil to protect from light. The samples were extracted with methanol multiple times. The supernatant was filtered with Whatman filter paper No1. Two-volumes of $15 \%$ sodium chloride was added to the methanol extract and after mixing equal volume of hexane was added. The yellow carotenoids were separated from the methanol-salt mix and accumulated in the hexane fraction. The hexane fraction was removed and washed at least three times with water. The hexane fraction was evaporated and the resultant carotenoid pellet was dissolved in methanol. 


\section{Supplementary Figure 9: Spectrophotometric analysis of methanolic extracts}

Methanolic extracts from K. rhizophila wildtype, crtEb(e17), crtl(e10), crtb(e6), crtYf(e18), and crtYe(e22) were analyzed at 350, 400, 450, 500, and $550 \mathrm{~nm}$ wavelengths. The absorbance of the extracts at each wavelength was plotted.

\section{Supplementary Figure 10: LC-MS analysis of carotenoid extracts from K. rhizophila mutants}

A) Chromatograms of absorbance at $440 \mathrm{~nm}$ during reverse phase LC separation of clarified extracts on a C18 column using an elution gradient from $2 \%$ to $50 \%$ MTBE. Traces from extracts of Wildtype (gray), crtYe(e22) (blue), and crtEb(e17) (red) are overlaid.

B) Absorbance spectra at the indicated elution times from crtEb(e17) (reds) or crtYe(e22) (blue) chromatograms, with absorbance peaks indicated. At least two compounds coelute in the main peak $\sim 18$ min with distinct absorbance spectra, as shown, on the peak (18.1 $\mathrm{min})$ and shoulder (18.5 $\mathrm{min})$.

C) MS spectral identification of a compound eluting at $\sim 18$ min with chemical formula $\mathrm{C} 4 \mathrm{OH} 56$, consistent with lycopene, in the extracts from both mutants, as well as the calculated isotopic distribution for the identified ion (red bars).

\section{Supplementary Figure 11: Antioxidants did not suppress pgp-5p::gfp induction in eft-}

\section{3(q145); pgp-5p::gfp animals}

A) N-Acetyl Cysteine, Ascorbic acid, Trolox or Resveratrol did not suppress pgp-5p::gfp in eft-3(q145); pgp-5p::gfp animals. Synchronized eft-3(q145); pgp-5p::gfp animals were grown from L1-larval stage in the presence of N-Acetyl Cysteine, Ascorbic acid, Trolox or Resveratrol and imaged after 50 hours at $20^{\circ} \mathrm{C}$.

B) Beta-carotene or Astaxanthin did not suppress pgp-5p::gfp in eft-3(q145); pgp-5p::gfp animals. Synchronized eft-3(q145); pgp-5p:::gfp animals were grown from L1-larval stage in the presence of Beta-carotene or Astaxanthin and imaged after 50 hours at $20^{\circ} \mathrm{C}$. 
C) E. coli expressing either zeaxanthin, neurosporene, violaxanthin, delta-carotene, or alpha-carotene did not suppress pgp-5p::gfp in eft-3(q145); pgp-5p::gfp animals.

D) $p g p-5 p:: g f p$ induction in response to $10 \mu \mathrm{g} / \mathrm{ml}$ and $20 \mu \mathrm{g} / \mathrm{ml}$ of hygromycin was significantly reduced in animals fed on K. rhizophila wildtype. The pgp-5p::gfp induction in response to $50 \mu \mathrm{g} / \mathrm{ml}$ hygromycin is normal in animals fed on E. coli OP50 or on K. rhizophila wildtype.

\section{Supplementary Figure 12: K. rhizophila carotenoid sensitizes the animals to toxins affecting protein synthesis but not to mitochondrial toxin}

A) $p g p-5 p:: g f p$ induction in response to $2.5 \mu \mathrm{g} / \mathrm{ml}$ or $6.25 \mu \mathrm{g} / \mathrm{ml}$ of emetine was significantly reduced in animals fed on $K$. rhizophila wildtype. However, in animals treated with $12.5 \mu \mathrm{g} / \mathrm{ml}$ of emetine, pgp-5p::gfp induction was partially reduced in animals fed on $K$. rhizophila. In animals treated with $25 \mu \mathrm{g} / \mathrm{ml}$ of emetine, the induction of pgp-5p::gfp was normal both animals treated with either E. coli OP50 or K. rhizophila wildtype.

B) $p g p-5 p:: g f p$ induction in response to $6.25 \mu \mathrm{g} / \mathrm{ml}$ of emetine was significantly reduced in animals fed on $K$. rhizophila wildtype while mutants in $K$. rhizophila crtEb(e17) or $K$. rhizophila crtl(e10) did not suppress the GFP induction

C) Animals treated with $K$. rhizophila carotenoid extract were hypersensitive to Hygromycin. Synchronized wildtype animals treated with solvent control or $K$. rhizophila extract from L1-larval stage were exposed to hygromycin at L3-larval stage and scored for development 60 hours later at $20^{\circ} \mathrm{C}$. Animals treated with K. rhizophila extract and hygromycin appear smaller compared to animals on solvent control and hygromycin.

D) $p g p-5 p:: g f p$ induction in response to $1 \mathrm{mM}$ of cisplatin was significantly reduced in animals fed on K. rhizophila wildtype while mutants in K. rhizophila crtEb(e17), crtYe(e22) or crtl(e10) did not suppress the GFP induction. Synchronized pgp-5p::gfp animals were grown on E. coli OP50 plates from L1-larval stage and transferred to either K. rhizophila wildtype or K. rhizophila crtEb(e17), crtYe(e22) or crtl(e10) at L4stage at $20^{\circ} \mathrm{C}$. After 24 hours, the animals were treated with either solvent or cisplatin and scored for induction of GFP after 24 hours.

E) Animals fed on control extract or $K$. rhizophila extract were sensitive to antimycin. Synchronized wildtype animals treated with solvent control or K. rhizophila extract from 
bioRxiv preprint doi: https://doi.org/10.1101/2020.01.08.898668; this version posted January 9,2020 . The copyright holder for this preprint

(which was not certified by peer review) is the author/funder, who has granted bioRxiv a license to display the preprint in perpetuity. It is made available under aCC-BY 4.0 International license.

L1-larval stage were exposed to antimycin at L3-larval stage and scored for development 60 hours later at $20^{\circ} \mathrm{C}$.

\section{Supplementary Table 1}

Analysis of the effects of $K$. rhizophila mutants on gfp induction in eft-3(q145); pgp5p::gfp animals and pgp-5p::gfp animals. 


\section{Materials and methods}

N2 Bristol was the wildtype strain used.

The following strains and mutant alleles were used:

SJ4100 [zc/s13[hsp-6::GFP], WE5172 [ajls1(pgp-5::gfp)X], JG16 [eft-3(q145)/hT2[bli-4(e937) let-?(q782) qls48] (I;III); ajls1(pgp-5::gfp)X], JG20 [ajls1(pgp-5::gfp); agEX(pvha-6::mcherry::zip-2+ myo-2::gfp)],

AY101 [acls101[F35E12.5P::GFP + rol-6(su1006)]

SJ4005 [zcls4 [hsp-4::GFP] V], SJ4100(zcls13[hsp-6::GFP])

\section{Growth and handling of microbes used:}

$16 S$ ribosomal sequence was amplified using specific primers and sequenced to identify the microbes. LB media as well as plates was used for culturing Kocuria rhizophila, Arthrobacter arilaitensis and Corynebacterium glutamicum and its mutants. $500 \mu$ of overnight culture was seeded onto SK media plates and incubated at room temperature for 2 days before initiating the experiments. For experiments involving Kocuria rhizophila wildtype and mutants, Arthrobacter arilaitensis, and Corynebacterium glutamicum wildtype and as well as mutants, synchronized L1-larval stage animals grown until L4-larval stage or day one of adulthood in E. coli OP50 seeded plates and was washed in M9 buffer at least five times before transferring to the appropriate bacterial food.

\section{Drug treatments:}

Hygromycin diluted in M9 solution to the desired concentration was added onto preseeded E.coli OP50 bacteria containing NGM plates. Stock solution of emetine or cisplatin was diluted in M9 and the desired concentration was added onto preseeded E.coli OP50 bacteria containing NGM plates. $750 \mu \mathrm{g} / \mathrm{ml}$ of $K$. rhizophila extracts was added onto preseeded E.coli OP50 bacteria containing NGM plates containing appropriate concentrations of hygromycin 
or cisplatin or emetine. For the xenobiotic experiments, synchronized L1-stage animals were dropped onto the drug containing plates and scored 4 days later.

Food aversion assay was performed by adding either hygromycin or cisplatin to $E$. coli OP50 lawns that contained synchronized L4 larval stage worms that were treated with $750 \mu \mathrm{g} / \mathrm{ml}$ of $K$. rhizophila extracts. Aversion index was calculated by counting number of animals off lawn over number of total animls counted at desired time point (Aversion index $=\mathrm{N}_{\text {(animals off lawn) }} / \mathrm{N}_{\text {(total animals). }}$ Each experiment was conducted in triplicate and the experiments were repeated in three independent trials.

\section{RNAi Assays:}

For RNAi assays synchronized L1 larval stage animals of the appropriate genotype were fed with appropriate RNAi clones until they reach day one of adulthood. Subsequently, the RNAitreated animals were washed in M9 at least five times to remove the $E$. coli bacteria and transferred to K. rhizophila seeded plates or E. coli OP50 seeded plates.

\section{Microscopy}

Nematodes were mounted onto agar pads and images were acquired using a Zeiss AXIO Imager Z1 microscope fitted with a Zeiss AxioCam HRm camera and Axiovision 4.6 (Zeiss) software. All the fluorescence images shown within the same figure panel were collected together using the same exposure time. Images were converted to 8-bit image, thresholded and quantified using ImageJ. Student's t test was used determine statistical significance. Low-magnification bright-field and GFP fluorescence images were acquired using a Zeiss AxioZoom V16, equipped with a Hammamatsu Orca flash 4.0 digital camera, and using Axiovision ZEN software.

\section{Multiple alignment of protein sequences}

Multiple alignments were performed using Clustal Omega:

\section{(https://www.ebi.ac.uk/Tools/msa/clustalo/)}




\section{K. rhizophila EMS mutagenesis screen}

Mutagenesis was performed by treatment of overnight culture of $K$. rhizophila in PBS solution with $50 \mathrm{mM}$ EMS for 45 minutes at $37^{\circ} \mathrm{C}$. Serial dilutions of the mutagenized $\mathrm{K}$. rhizophila cultures were plated onto LB media plates and 2000 mutagenized bacterial colonies were picked and grown in LB solution. $500 \mu$ of overnight culture was seeded onto SK media plates and incubated at room temperature for two days before initiating the experiments. Synchronized L1-larval stage in eft-3(q145);pgp-5p::gfp animals grown until L4-larval stage or day one of adulthood in E. coli OP50 seeded plates and was washed in M9 buffer at least five times before transferring to the $K$. rhizophila mutant bacterial food. The plates were visually screened after 24 hours for GFP induction.

\section{Identification of EMS induced mutations by whole genome sequencing}

Genomic DNA extraction, library prep, Illumina MiniSeq sequencing and bioinformatics were all performed by The Sequencing Center (www.thesequencingcenter.com). For genomic DNA isolation, The Sequencing Center used the Quick-DNA Fungal/Bacterial Miniprep Kit, a standard DNA extraction kit that uses bead beating methods to dissolve cell walls without organic denaturants or proteinases. The Illumina Nextera XT DNA Library Prep kit and protocol were used for library preparation. DNA quantitation was performed on a Qubit Fluorometer. The Illumina MiniSeq System Denature and Dilute Libraries Guide was followed to dilute and denature the final library pool, including a 1\% PhiX spike-in control before loading onto the sequencer. The final library was loaded onto an Illumina High Output Reagent Kit on an Illumina MiniSeq with $2 \times 150$ paired-end reads. Sequencing data was processed through a Geneious Prime bioinformatics pipeline. Sequencing reads were 
aligned to the Kocuria rhizophila DC2201 (NC_010617.1) reference genome to identify mutant alleles and gene variants.

\section{Isolation of $K$. rhizophila carotenoids}

Carotenoid isolation from K. rhizophila was isolated as described ${ }^{1}$ with the following modifications. K. rhizophila cultures grown in LB solution was washed with equal volume of water after centrifugation at 4000RPM for $15 \mathrm{~min}$. After centrifugation to remove water, equal volume of acetone was added and centrifuged again at 4000RPM for $15 \mathrm{~min}$. After removal of acetone, the bacterial pellets were extracted with methanol at $65^{\circ} \mathrm{C}$ in water bath after wrapping the samples with aluminum foil to protect from light. The samples were extracted with methanol multiple times. The supernatant was filtered with Whatman filter paper No1. Two-volumes of $15 \%$ sodium chloride was added to the methanol extract and after mixing equal volume of hexane was added. The yellow carotenoids were separated from the methanol-salt mix and accumulated in the hexane fraction. The hexane fraction was removed and washed at least three times with water. The hexane fraction was evaporated and the resultant carotenoid pellet was dissolved in methanol.

\section{High performance liquid chromatography}

Crude methanolic extracts were separated over an Agilent Eclipse Plus C18 4.6 x 250 mm column with a 5-micron particle size using an Agilent 1200 HPLC equipped with a diode array detector, autosampler, column oven, solvent degasser, and binary pump. The mobile phases were (A) water vs. (B) methanol at a flow rate of $2 \mathrm{~mL} / \mathrm{min}$. The column was preequilibrated at $40^{\circ} \mathrm{C}$ with $90 \%$ B prior to sample injection. Following injection, the column was washed isocratically for $5 \mathrm{~min}$ at $90 \% \mathrm{~B}$ before ramping to $100 \% \mathrm{~B}$ over $5 \mathrm{~min}$. Eluate absorbance spectra were monitored from $300-700 \mathrm{~nm}$.

\section{Liquid chromatography mass spectrometry (LC-MS)}


Methanol $(\mathrm{MeOH})$ extracts were diluted in dichloromethane (DCM) and filtered over prewashed silica gel using 15:85 methanol/DCM. The visibly colored eluate was collected, aliquoted, and dried under vacuum. Dry aliquots were stored under $\mathrm{Ar}$ at $-30^{\circ} \mathrm{C}$ and were resuspended just prior to analysis in a minimal volume of 1:9 water/MeOH. The material was separated over Waters XBridge C18 1 x $100 \mathrm{~mm}$ column with a 3.5-micron particle size at 25 ${ }^{\circ} \mathrm{C}$ using an Agilent 1200 HPLC equipped with a diode array detector, autosampler, column oven, solvent degasser, and binary pump. The mobile phases were (A) LC-MS grade 1:9 water/MeOH vs. (B) HPLC-grade methyl tert-butyl ether (MTBE) at a flow rate of $150 \mu \mathrm{L} / \mathrm{min}$. The column was preequilibrated with $2 \%$ B prior to sample injection. Following injection, the column was washed isocratically for $5 \mathrm{~min}$ at $2 \% \mathrm{~B}$ before ramping to $30 \% \mathrm{~B}$ over $25 \mathrm{~min}$. Eluate absorbance spectra were monitored from 225-650 nm, and high-resolution mass analysis was performed on-line with an Agilent 6230 TOF mass spectrometer equipped with a Multimode source in positive atmospheric pressure chemical ionization (APCI+) mode. The MS source parameters were as follows: nitrogen drying gas temperature, $325^{\circ} \mathrm{C}$; drying gas flow, $5 \mathrm{~L} / \mathrm{min}$; vaporizer temperature, $200^{\circ} \mathrm{C}$; charging voltage, $2000 \mathrm{~V}$; capillary voltage, $2000 \mathrm{~V}$; nebulizer pressure, 25 psig; and corona current, $4 \mu \mathrm{A}$. The fragmentor voltage was set to $250 \mathrm{~V}$, and MS1 scans were acquired from $100-3200 \mathrm{~m} / \mathrm{z}$ at a rate of $1 \mathrm{~Hz}$. Compound peaks were identified by searching high resolution mass spectra by chemical formula from a database of known carotenoids, using a stringent mass error threshold (5 ppm), and then correlating extracted ion chromatograms (EIC) of candidate hits to $440 \mathrm{~nm}$ absorbance elution peak profiles.

\section{Putative "decaprenoxanthin" carotenoid biosynthetic cluster from microbes}

Putative carotenoid biosynthetic cluster of Leifsonia xyli (Lxx15630, Lxx15620, Lxx15610, Lxx15600, Lxx15590, and Lxx15580) ${ }^{3}$, Microbacterium testaceum (MTES_3133, 
MTES_3132, MTES_3131, MTES_3130, MTES_3129, and MTES_3128) ${ }^{4}$, Cellvibrio gilvus

(Celgi_1516, Celgi_1515, Celgi_1514, Celgi_1513, Celgi_1512, and Celgi_1511)

Cellulomonas fimi (Celf_3171, Celf_3170, Celf_3169, Celf_3168, Celf_3167, and

Celf_3166) ${ }^{5}$, Sanguibacter keddieii (Sked_12750, Sked_12760, Sked_12770, Sked_12780,

Sked_12790, and Sked_12800) ${ }^{6}$, Jonesia denitrificans (Jden_0342, Jden_0341, Jden_0340,

Jden_0339, Jden_0338, and Jden_0337)7, Mycetocola manganoxydans (D9V29_RS08865,

D9V29_RS08870, D9V29_RS08875, D9V29_RS08880, D9V29_RS08885, and

D9V29_RS08890), Mycetocola miduiensis (BM197_RS02470, BM197_RS02475,

BM197_RS02480, BM197_RS02485, BM197_RS02490, and BM197_RS02495),

Cryobacterium psychrotolerans (BLQ39_RS02180, BLQ39_RS02185, BLQ39_RS02190,

BLQ39_RS02195, BLQ39_RS02200, and BLQ39_RS02205), Subtercola boreus

(B7R21_RS02695, B7R21_RS02700, B7R21_RS02705, B7R21_RS02710,

B7R21_RS02715, and B7R21_RS02720), Herbiconiux solani (HSO01S_RS07000,

HS001S_RS07005, HS001S_RS07010, HSO01S_RS07015, HSO01S_RS07020, and

HSO01S_RS07025), Microbacterium phyllosphaerae (D3H67_RS09120, D3H67_RS09125,

D3H67_RS09130, D3H67_RS09135, D3H67_RS09140, and D3H67_RS09145), Leifsonia

aquatica (N136_RS22055, N136_RS22060, N136_RS22065, N136_RS22070,

N136_RS22075, and N136_RS22080), Microbacterium esteraromaticum (B4U78_RS09520,

B4U78_RS09525, B4U78_RS09530, B4U78_RS09535, B4U78_RS09540, and

B4U78_RS09545),

Plantibacter sp. H53 (A4X17_RS18565, A4X17_RS18570, A4X17_RS18575,

A4X17_RS18580, A4X17_RS18585, and A4X17_RS18590), Curtobacterium sp.

(ASF23_RS14315, ASF23_RS14320, ASF23_RS14325, ASF23_RS14330,

ASF23_RS14335, and ASF23_RS14340), Microterricola pindariensis (GY24_RS04745,

GY24_RS04750, GY24_RS04755, GY24_RS04760, GY24_RS04765, and GY24_RS04770), 
Frondihabitans sp. (EDF46_RS08000, EDF46_RS08005, EDF46_RS08010, EDF46_RS08015, EDF46_RS08020, and EDF46_RS08025), Salinibacterium xinjiangense (SAMN06296378_0676, SAMN06296378_0677, SAMN06296378_0678, SAMN06296378_0679, SAMN06296378_0680, and SAMN06296378_0681), Agromyces sp. (AVP42_RS01110, AVP42_RS01115, AVP42_RS01120, AVP42_RS01125, AVP42_RS01130, and AVP42_RS01135), Microbacterium barkeri (MBR4_RS00310, MBR4_RS00315, MBR4_RS00320, MBR4_RS00325, MBR4_RS00330, and MBR4_RS00335), Arthrobacter koreensis (BN2404_RS04370, BN2404_RS04375, BN2404_RS04380, BN2404_RS04385, BN2404_RS04390, and BN2404_RS04395), Cryobacterium roopkundense (GY21_RS00565, GY21_RS00570, GY21_RS00575, GY21_RS00580, GY21_RS00585, and GY21_RS00590), Microbacterium oxydans (RN51_RS07325, RN51_RS07330, RN51_RS07335, RN51_RS07340, RN51_RS07345, and RN51_RS07350), Arthrobacter luteolus (AL3_RS02570, AL3_RS02575, AL3_RS02580, AL3_RS02585, AL3_RS02590, and AL3_RS02595), Cryobacterium aureum (CJ028_RS03575, CJ028_RS03580, CJ028_RS03585, CJ028_RS03590, CJ028_RS03595, and CJ028_RS03600), Curtobacterium ammoniigenes (CAM01S_RS13455, CAM01S_RS13460, CAM01S_RS13465, CAM01S_RS13470, CAM01S_RS13475, and CAM01S_RS13480), Oerskovia enterophila (OJAG_RS08920, OJAG_RS08925, OJAG_RS08930, OJAG_RS08935,OJAG_RS08940, and OJAG_RS08945), Microbacterium paraoxydans (SAMN04489809_1122, SAMN04489809_1123, SAMN04489809_1124, SAMN04489809_1125, SAMN04489809_1126, and SAMN04489809_1127), Agromyces subbeticus (H521_RS21795, H521_RS21800, H521_RS0106365, H521_RS0106370, H521_RS21805, and H521_RS0106380), Arthrobacter crystallopoietes (D477_RS18370, D477_RS18375, D477_RS18380, D477_RS18385, and D477_RS18390, D477_RS18395), Georgenia satyanarayanai 
(DSZ44_RS04745, DSZ44_RS04750, DSZ44_RS04755, DSZ44_RS04760, DSZ44_RS04765, and DSZ44_RS04770), Microbacterium trichothecenolyticum (RS82_RS03115, RS82_RS03120, RS82_RS03125, RS82_RS03130, RS82_RS03135, and RS82_RS03140), Arthrobacter woluwensis (C6401_RS03950, C6401_RS03955, C6401_RS03960, C6401_RS03965, C6401_RS03970, and C6401_RS03975), Promicromonospora kroppenstedtii (PROKR_RS13815, PROKR_RS13820, PROKR_RS13825, PROKR_RS13830, PROKR_RS13835, and PROKR_RS13840), Cellulomonas cellasea (Q760_RS04595, Q760_RS04600, Q760_RS04605, Q760_RS18340, Q760_RS04615, and Q760_RS04620), Agromyces cerinus (BUR99_RS12060, BUR99_RS12065, BUR99_RS12070, BUR99_RS12075, BUR99_RS12080, and BUR99_RS12085), Agreia pratensis (B9Y86_RS06900, B9Y86_RS06905, B9Y86_RS06910, B9Y86_RS06915, B9Y86_RS06920 and B9Y86_RS06925), Microbacterium laevaniformans (OR221_3062, OR221_3063, OR221_3064, OR221_3065, OR221_3066, and OR221_3067), Arthrobacter stackebrandtii (CVV67_17780, CVV67_17785, CVV67_17790, CVV67_17795, CVV67_17800, and CVV67_17805), Paeniglutamicibacter gangotriensis (ADIAG_RS03760, ADIAG_RS03765, ADIAG_RS03770, ADIAG_RS03775, ADIAG_RS03780, and ADIAG_RS03785), Microbacterium trichothecenolyticum (RS82_RS03115, RS82_RS03120, RS82_RS03125, RS82_RS03130, RS82_RS03135, and RS82_RS03140), Arthrobacter livingstonensis (CVV68_RS19330, CVV68_RS19335, CVV68_RS19340, CVV68_RS19345, CVV68_RS19350, and CVV68_RS19355), Demequina lutea (AOP76_RS09030, AOP76_RS09035, AOP76_RS09040, AOP76_RS09045, AOP76_RS09050, and AOP76_RS09055), Zhihengliuella halotolerans (CUR88_RS12685, CUR88_RS12690, CUR88_RS12695, CUR88_RS12700,CUR88_RS12705, and CUR88_RS12710), Paeniglutamicibacter antarcticus (BN2261_RS08280, BN2261_RS08285, BN2261_RS08290, BN2261_RS08295, BN2261_RS08300, and BN2261_RS08305), 
Janibacter melonis (EEW87_RS00715, EEW87_RS00720, EEW87_RS00725, EEW87_RS00730, EEW87_RS00735, and EEW87_RS00740), Microbacterium arborescens (DOU46_RS02280, DOU46_RS02285, DOU46_RS02290, DOU46_RS02295, DOU46_RS02300, and DOU46_RS02305), Agreia pratensis (B9Y86_RS06900, B9Y86_RS06905, B9Y86_RS06910, B9Y86_RS06915, B9Y86_RS06920, and B9Y86_RS06925), Agreia bicolorata (TZ00_RS04480, TZ00_RS04485, TZ00_RS19215, TZ00_RS04495, TZ00_RS04500, and TZ00_RS04505), Arthrobacter psychrochitiniphilus (CVS30_RS02785, CVS30_RS02790, CVS30_RS02795, CVS30_RS02800, CVS30_RS02805, and CVS30_RS02810), Microterricola pindariensis (GY24_RS04745, GY24_RS04750, GY24_RS04755, GY24_RS04760, GY24_RS04765, and GY24_RS04770), Microbacterium indicum (H576_RS15860, H576_RS0112930, H576_RS0112935, H576_RS0112940, H576_RS0112945, and H576_RS15865), Homoserinimonas sp. (DL891_RS01870, DL891_RS01875, DL891_RS01880, DL891_RS01885, DL891_RS01890, and DL891_RS01895), Cryobacterium levicorallinum (SAMN05216274_11068, SAMN05216274_11069, SAMN05216274_11070, SAMN05216274_11071, SAMN05216274_11072, and SAMN05216274_11073), Frigoribacterium sp.(EDF18_RS14355, EDF18_RS14360, crtl, EDF18_RS14370, EDF18_RS14375, and EDF18_RS14380), Cryobacterium luteum (SAMN05216281_10883, SAMN05216281_10884, SAMN05216281_10885, SAMN05216281_10886, SAMN05216281_10887, and SAMN05216281_10888), Cellulomonas carbonis (N868_RS13600, N868_RS13605, N868_RS13610, N868_RS13615, N868_RS13620, and N868_RS13625), Okibacterium fritillariae (B5X75_RS14075, B5X75_RS14080, B5X75_RS14085, B5X75_RS14090, B5X75_RS14095, and B5X75_RS14100), Glycomyces sambucus (BLS99_RS13650, BLS99_RS13655, BLS99_RS13660, BLS99_RS13665, BLS99_RS13670, and BLS99_RS13675), Krasilnikoviella flava (B5Y66_RS20515, B5Y66_RS20520, 
B5Y66_RS20525, B5Y66_RS20530, B5Y66_RS20535, and B5Y66_RS20540), Actinotalea ferrariae (N866_01505, N866_01510, N866_01515, N866_01520, N866_01525, and ubiA), Lysinimicrobium soli (AOM04_RS11780,AOM04_RS11785, AOM04_RS11790, AOM04_RS11795, AOM04_RS11800, and AOM04_RS11805), Luteimicrobium subarcticum (CLV34_RS08275, CLV34_RS08280, CLV34_RS08285, CLV34_RS08290, CLV34_RS08295, and CLV34_RS08300), Promicromonospora kroppenstedtii (PROKR_RS13815, PROKR_RS13820, PROKR_RS13825, PROKR_RS13830, PROKR_RS13835, and PROKR_RS13840), Tersicoccus phoenicis (BKD30_RS05860, BKD30_RS05865, BKD30_RS05870, BKD30_RS05875, BKD30_RS05880, and BKD30_RS05885), Sinomonas humi (LK10_RS09295, LK10_RS09300, LK10_RS09305, LK10_RS09310, and LK10_RS09315), Pseudarthrobacter phenanthrenivorans (RM50_RS01675, RM50_RS01680, RM50_RS01685, RM50_RS01690, RM50_RS01695, and RM50_RS01700), Acaricomes phytoseiuli (C501_RS0107225, C501_RS0107230, C501_RS0107235, C501_RS0107240,C501_RS0107245,and C501_RS0107250), Leucobacter musarum (AMS67_RS10795, AMS67_RS10800, AMS67_RS10805, AMS67_RS10810, AMS67_RS10815, and AMS67_RS10820), Ornithinimicrobium pekingense (K330_RS19765, K330_RS0107130, K330_RS19770, K330_RS19775, K330_RS0107145, and K330_RS0107150), Citricoccus sp. (CITRI_RS16000, CITRI_RS0102550,CITRI_RS0102555, CITRI_RS16005, CITRI_RS0102565, and CITRI_RS0102570) and Arthrobacter arilatensis (AARI_13710, AARI_13720, AARI_13730, AARI_13740, AARI_13760, and AARI_13750) ${ }^{8}$ are very similar have a similar size and show the same organization as in the genome of K. rhizophila. In Corynebacterium glutamicum (cg0723, cg0721, cg0720, cg0719, cg0718, and cg0717) $)^{9}$ and Corynebacterium efficiens (HMPREF0290_1086, HMPREF0290_1088, HMPREF0290_1089, HMPREF0290_1090, HMPREF0290_1091, and HMPREF0290_1092) ${ }^{10}$ the decaprenoxanthin producing gene 
cluster is similar in size and organization to that of $K$. rhizophila except for the insertion of an unrelated gene cg0722 between crtE and crtB in C. glutamicum or HMPREF0290_1088 in C. efficiens. Also, in Corynebacterium glutamicum, an additional carotenoid cluster (NCgI0600, NCgl0598, NCgl0597, NCgl0596, NCgl0595, and NCgl0594) in also present in the genome. In Kytococcus sedentarius, the genes responsible for carotenoid production (Ksed_13840, Ksed_13830, Ksed_13820, Ksed_13810, Ksed_13800) are arranged in the same cluster while Ksed_16070, which encodes for geranylgeranyl pyrophosphate synthase is located elsewhere in the genome ${ }^{11}$.

In Brevibacterium mcbrellneri, the genes responsible for carotenoid production (HMPREF0183_0793, HMPREF0183_0794, HMPREF0183_0795, HMPREF0183_0796, and HMPREF0183_0797) are located in the same cluster while HMPREF0183_0437, which encodes for polyprenyl synthetase is located elsewhere in the genome.

In Beutenbergia cavernae, the genes responsible for carotenoid production (Bcav_3492, Bcav_3491, Bcav_3490, Bcav_3489, Bcav_3488) are located in the same cluster while Bcav_0970, which encodes for polyprenyl synthetase is located elsewhere in the genome ${ }^{12}$. In Brachybacterium faecium (Bfae_04470, Bfae_04440, Bfae_04430, Bfae_04420, Bfae_04410, and Bfae_04400) ${ }^{13}$ the carotenoid biosynthetic cluster is similar in size and organization to that of $K$. rhizophila except for the insertion of two unrelated genes Bfae_04460 and Bfae_04450 between Bfae_04470 and Bfae_04440. In Cellulomonas flavigna (Cfla_2888, Cfla_2889, Cfla_2890, Cfla_2891, and Cfla_2892) ${ }^{14}$ all the genes responsible for carotenoid production are present except for Cfla_2893 which is a likely pseudogene because of frame-shift mutation.

Decaprenoxanthin was the first C50 carotenoid discovered from Flavobacterium dehydrogenans (now known as Agromyces mediolanus ${ }^{15}$ ). 
Many bacteria including Agromyces mediolanus ${ }^{15}$, Aureobacterium sp. ${ }^{16}$, Arthrobacter glacialis $^{17}$, Arthrobacter arilatensis ${ }^{18}$, Cellulomonas biazotea ${ }^{19}$, and Corynebacterium glutamicum ${ }^{20}$ are known to produce decaprenoxanthin.

\section{Extended Reference:}

1. Giuffrida, D. et al. Characterisation of the $\mathrm{C} 50$ carotenoids produced by strains of the cheese-ripening bacterium Arthrobacter arilaitensis. International Dairy Journal 55, 1016 (2016).

2. Pilbrow, J., Sabherwal, M., Garama, D. \& Carne, A. A novel fatty acid-binding proteinlike carotenoid-binding protein from the gonad of the New Zealand sea urchin Evechinus chloroticus. PLOS ONE 9, e106465 (2014).

3. Monteiro-Vitorello, C. B. et al. The genome sequence of the gram-positive sugarcane pathogen Leifsonia xyli subsp. xyli. Mol. Plant Microbe Interact. 17, 827-836 (2004).

4. Morohoshi, T., Wang, W.-Z., Someya, N. \& Ikeda, T. Genome sequence of Microbacterium testaceum StLB037, an N-acylhomoserine lactone-degrading bacterium isolated from potato leaves. J Bacteriol 193, 2072-2073 (2011).

5. Christopherson, M. R. et al. The genome sequences of Cellulomonas fimi and "Cellvibrio gilvus" reveal the cellulolytic strategies of two facultative anaerobes, transfer of 'Cellvibrio gilvus' to the genus Cellulomonas, and proposal of Cellulomonas gilvus sp. nov. PLoS ONE 8, e53954 (2013).

6. Ivanova, N. et al. Complete genome sequence of Sanguibacter keddieii type strain (ST74). Stand Genomic Sci 1, 110-118 (2009).

7. Pukall, R. et al. Complete genome sequence of Jonesia denitrificans type strain (Prevot 55134). Stand Genomic Sci 1, 262-269 (2009).

8. Monnet, C. et al. The arthrobacter arilaitensis Re117 genome sequence reveals its genetic adaptation to the surface of cheese. PLOS ONE 5, e15489 (2010).

9. Kalinowski, J. et al. The complete Corynebacterium glutamicum ATCC 13032 genome sequence and its impact on the production of L-aspartate-derived amino acids and vitamins. J. Biotechnol. 104, 5-25 (2003).

10. Nishio, Y. et al. Comparative complete genome sequence analysis of the amino acid replacements responsible for the thermostability of Corynebacterium efficiens. Genome Res. 13, 1572-1579 (2003).

11. Sims, D. et al. Complete genome sequence of Kytococcus sedentarius type strain (541). Stand Genomic Sci 1, 12-20 (2009).

12. Land, M. et al. Complete genome sequence of Beutenbergia cavernae type strain (HKI 0122). Stand Genomic Sci 1, 21-28 (2009).

13. Lapidus, A. et al. Complete genome sequence of Brachybacterium faecium type strain (Schefferle 6-10). Stand Genomic Sci 1, 3-11 (2009).

14. Abt, B. et al. Complete genome sequence of Cellulomonas flavigena type strain (134). Stand Genomic Sci 3, 15-25 (2010).

15. Liaaen-Jensen, S., Hertzberg, S., Weeks, O. B. \& Schwieter, U. Bacterial carotenoids XXVII. C50-carotenoids. 3. Structure determination of dehydrogenans-P439. Acta Chem Scand 22, 1171-1186 (1968).

16. Fukuoka, S., Ajiki, Y., Ohga, T., Kawanami, Y. \& Izumori, K. Production of dihydroxy C50-carotenoid by Aureobacterium sp. FERM P-18698. Biosci. Biotechnol. Biochem. 68, 2646-2648 (2004). 
17. Arpin, N., Fiasson, J. L., Norgård, S., Borch, G. \& Liaaen-Jensen, S. Bacterial carotenoids, XLVI. C50-Carotenoids, 14. C50-Carotenoids from Arthrobacter glacialis. Acta Chem. Scand., B, Org. Chem. Biochem. 29, 921-926 (1975).

18. Sutthiwong, N. \& Dufossé, L. Production of carotenoids by Arthrobacter arilaitensis strains isolated from smear-ripened cheeses. FEMS Microbiol. Lett. 360, 174-181 (2014).

19. Weeks, O. B., Montes, A. R. \& Andrewes, A. G. Structure of the principal carotenoid pigment of Cellulomonas biazotea. J Bacteriol 141, 1272-1278 (1980).

20. Krubasik, P. et al. Detailed biosynthetic pathway to decaprenoxanthin diglucoside in Corynebacterium glutamicum and identification of novel intermediates. Arch. Microbiol. 176, 217-223 (2001). 
bioRxiv preprint doi: https://doi.org/10.1101/2020.01.08.898668; this version posted January 9,2020 . The copyright holder for this preprint (which was not certified by peer review) is the author/funder, who has granted bioRxiv a license to display the preprint in perpetuity. It is made available under aCC-BY 4.0 International license.

\section{Figure 1}

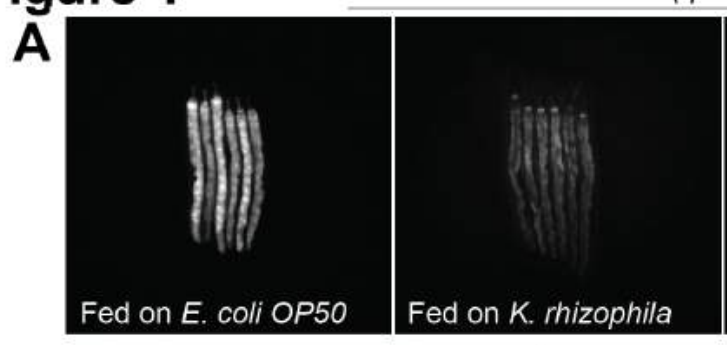

eft-3(q145); pgp-5p::gfp

B K. rhizophila crtYf(e18) -

K. rhizophila crtl(e10)

K. rhizophila crtEb(e17)

K. rhizophila wildtype

E. coli OP50
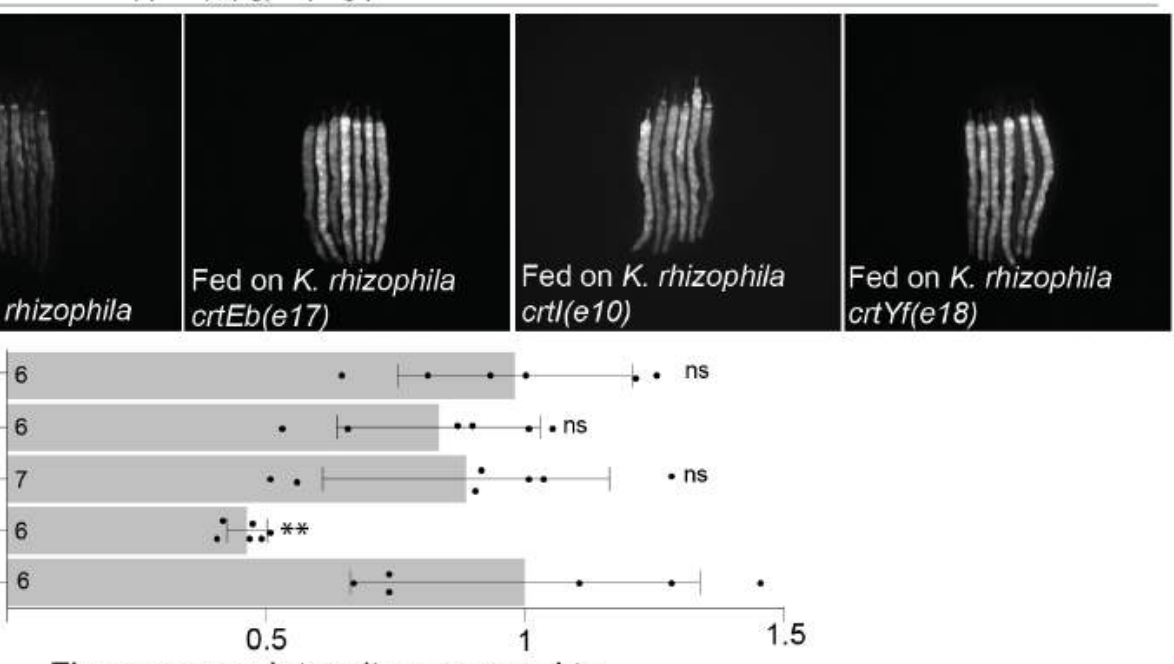

Fluorescence Intensity compared to eft-3(q145); pgp-5::gfp fed on E. coli OP50-1

C Isopentenylpyrophosphate (IPP), C5

GGPP Synthase (crtE) Dimethylallyl pyrophosphate (DMAPP), C5

Geranyl pyrophosphate (GPP), C10

GGPP Synthase (crtE) \اIPP, C5

Farnesylpyrophosphate (FPP), C15

GGPP Synthase (crtE) Џ IPP, C5

Geranylgeranyl pyrophosphate (GGPP), C20

Phytoene synthase (crtB) -GGPP, C20

Phytoene, C40

Phytoene desaturase (crtl)

Lycopene, C40

Lycopene elongase (crtEb)

Flavuxanthin, C50

carotenoid-e-cyclase (crtYe/f)

Decaprenoxanthin, C50
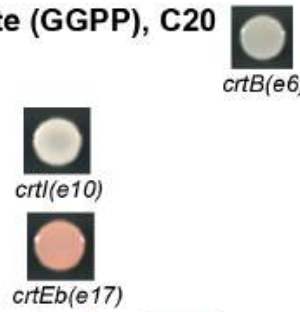

crtYe(e22) crtYf(e18)

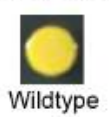


bioRxiv preprint doi: https://doi.org/10.1101/2020.01.08.898668; this version posted January 9, 2020. The copyright holder for this preprint (which was not certified by peer review) is the author/funder, who has granted bioRxiv a license to display the preprint in perpetuity. It is made available under aCC-BY 4.0 International license.

\section{Figure 2}

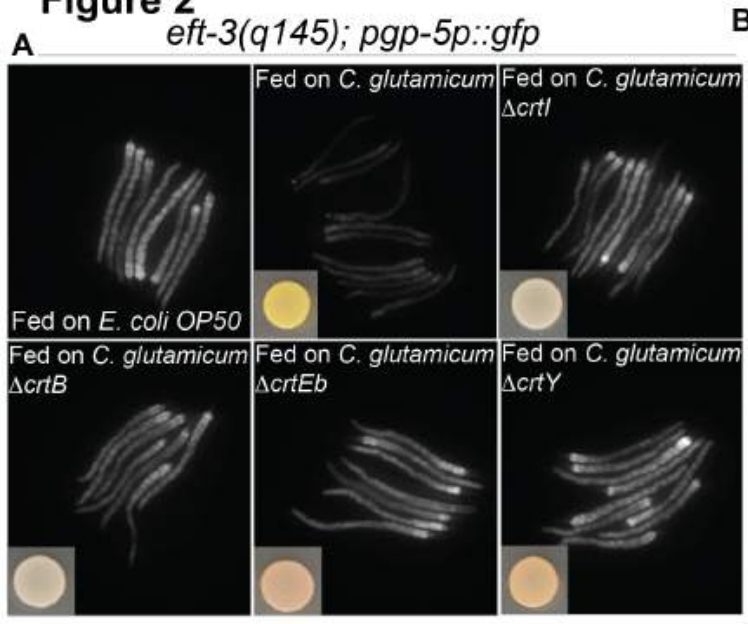

B

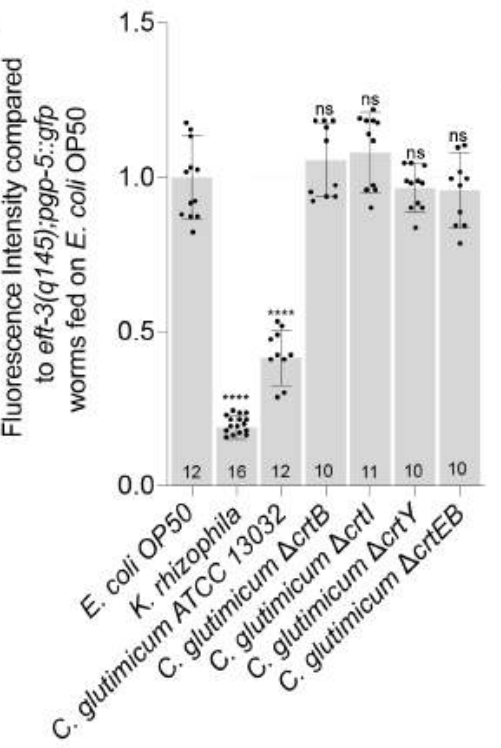

C

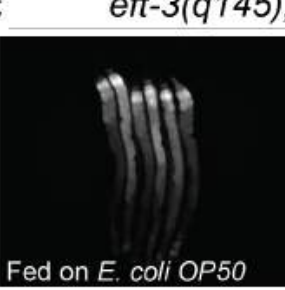

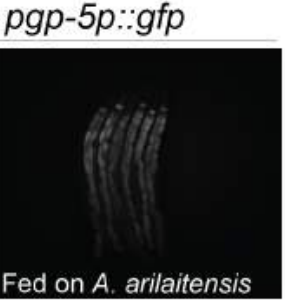


bioRxiv preprint doi: https://doi.org/10.1101/2020.01.08.898668; this version posted January 9, 2020. The copyright holder for this preprint (which was not certified by peer review) is the author/funder, who has granted bioRxiv a license to display the preprint in perpetuity. It is made available under aCC-BY 4.0 International license.

\section{Figure 3}

A

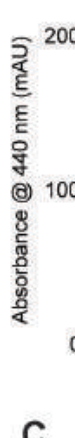

$\times 10^{3}$ Scan (it: $9.232-9.845 \mathrm{~min}, 38$ scans)

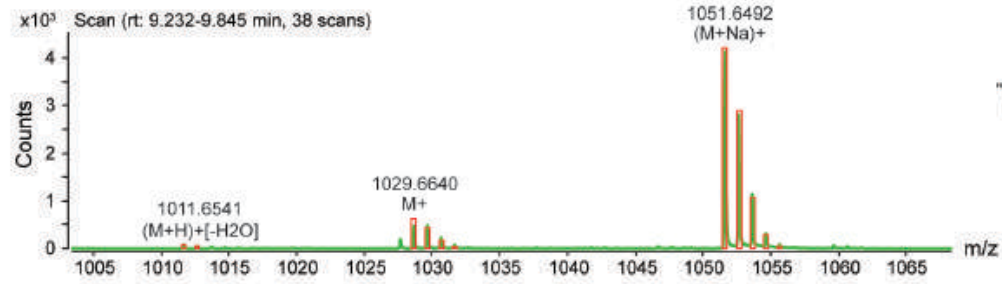

B

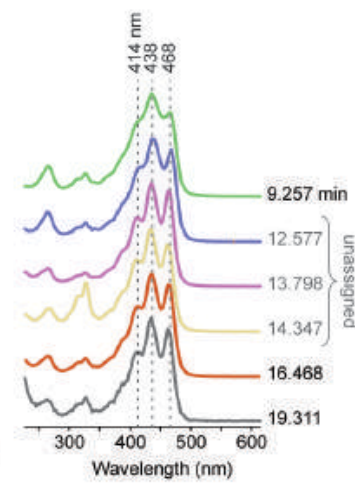

x10 Scan (it 16.304-16.999 min, 43 scans)
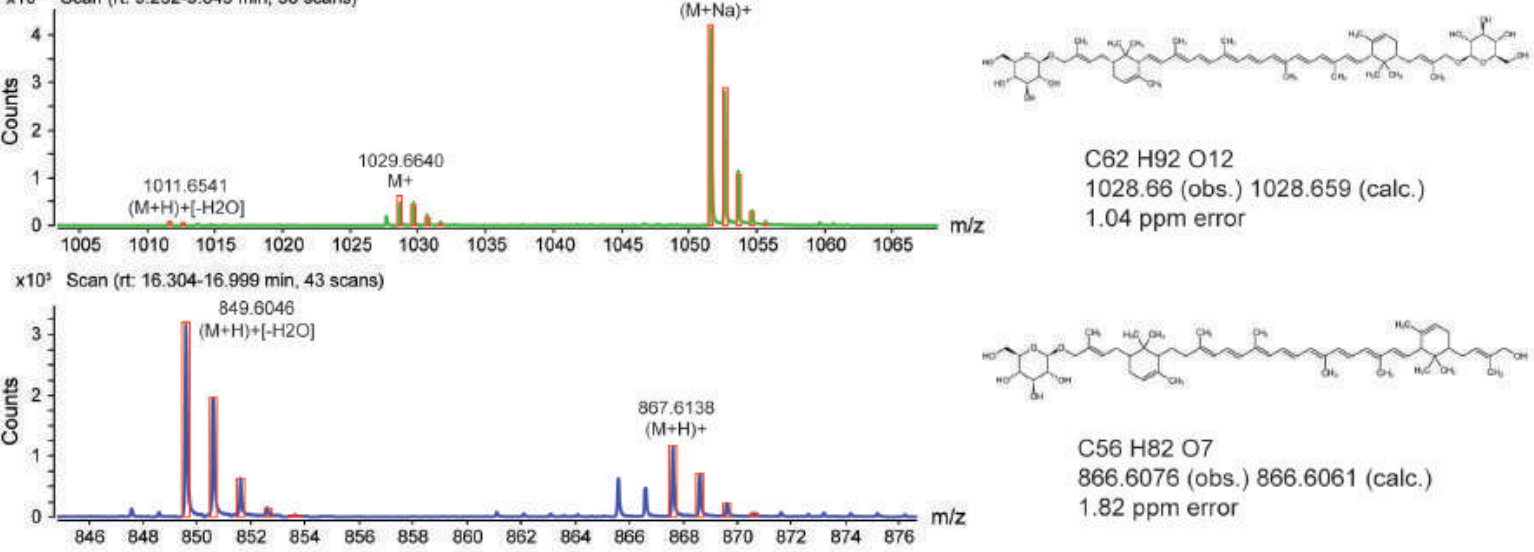

C62 H92 012

1028.66 (obs.) 1028.659 (calc.)

$1.04 \mathrm{ppm}$ error

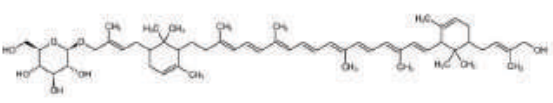

$\mathrm{C} 56 \mathrm{H} 82 \mathrm{O} 7$

866.6076 (obs.) 866.6061 (calc.)

$1.82 \mathrm{ppm}$ error

$\times 10^{3}$ Scan (it. 19.219-19.749 min, 33 scans)
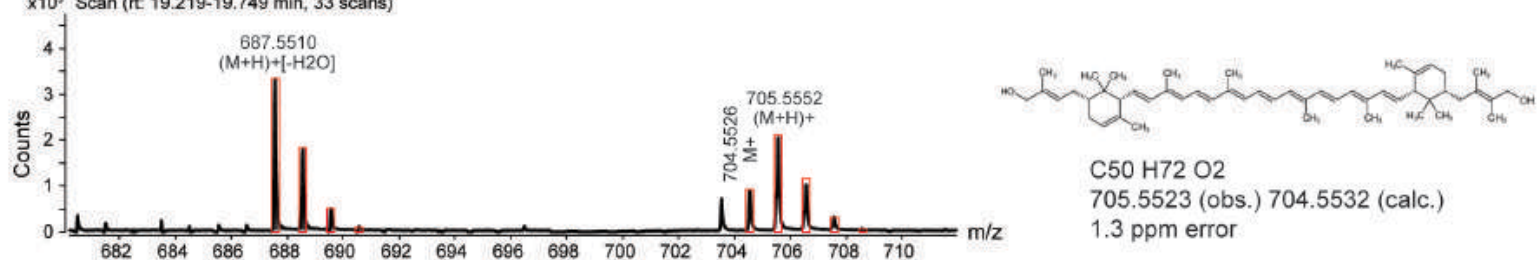

$\mathrm{C} 50 \mathrm{H} 72 \mathrm{O} 2$

705.5523 (obs.) 704.5532 (calc.)

$1.3 \mathrm{ppm}$ error

eft-3(q145);

D $\quad p g p-5 p:: g f p$

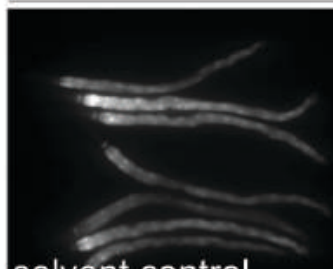

solvent control

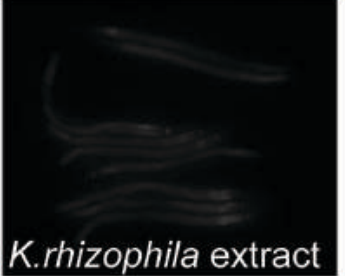




\section{Figure 4}
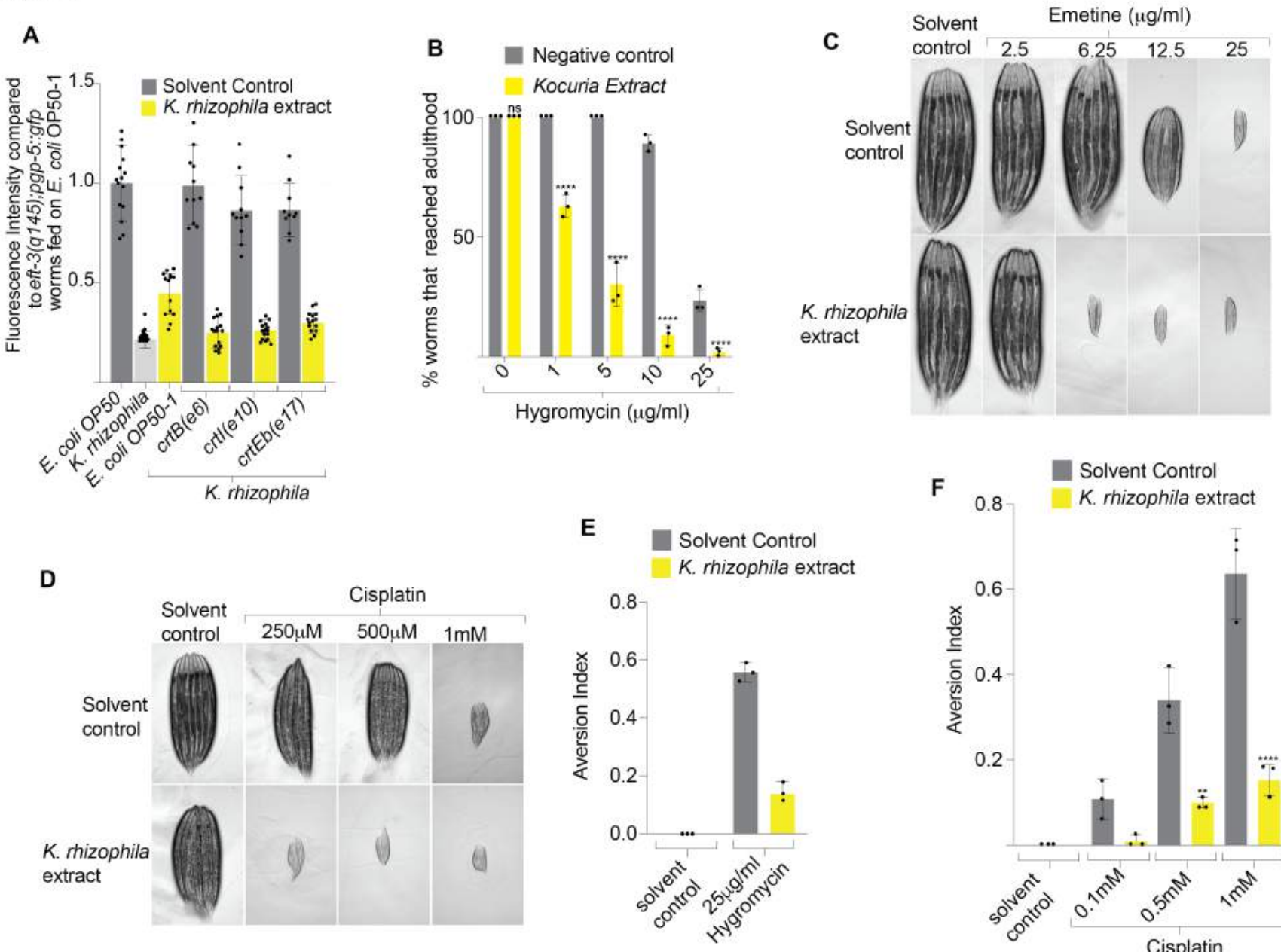
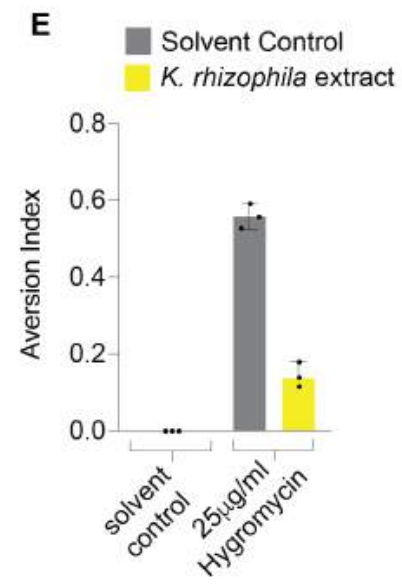

Solvent Control K. rhizophila extract

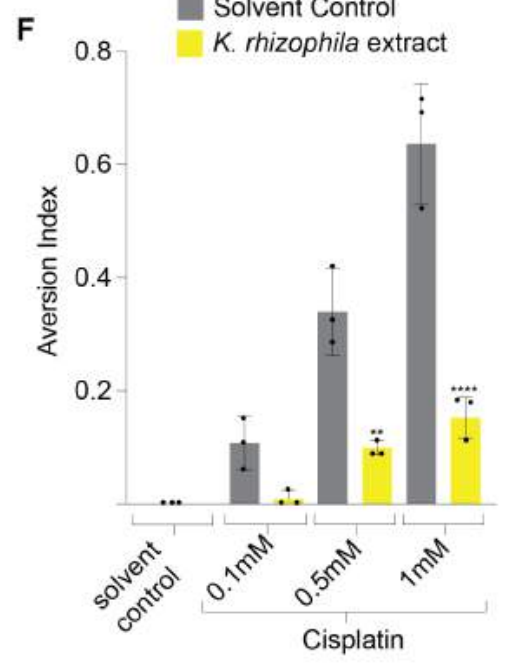


bioRxiv preprint doi: https://doi.org/10.1101/2020.01.08.898668; this version posted January 9, 2020. The copyright holder for this preprint (which was not certified by peer review) is the author/funder, who has granted bioRxiv a license to display the preprint in perpetuity. It is made available under aCC-BY 4.0 International license.

\section{Figure 5}

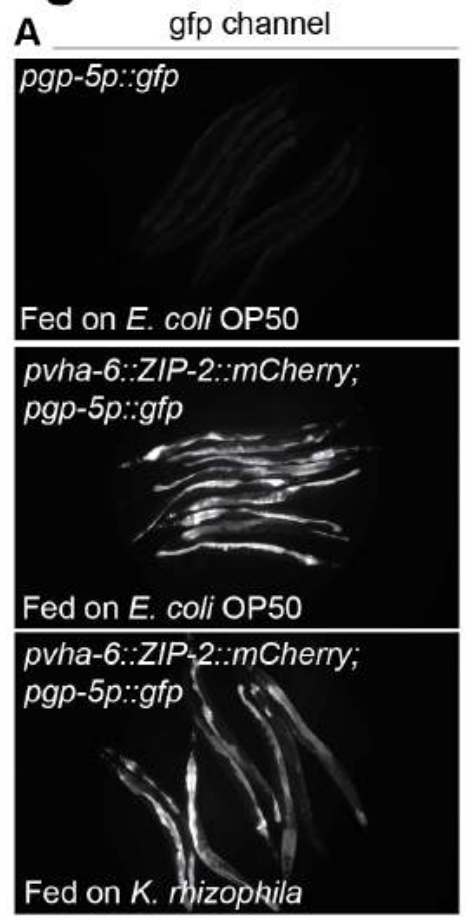

B

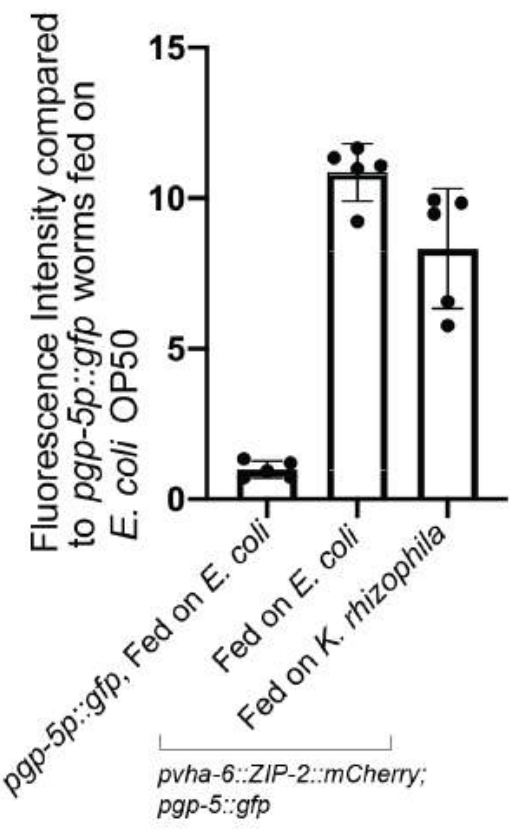

\section{C}
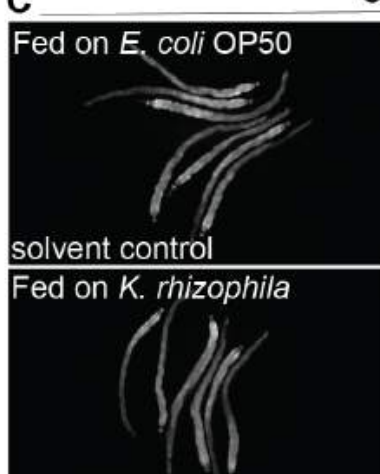

Cholic acid

Fed on K. rhizophila

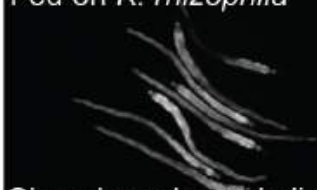

eft-3(q145); pgp-5p::gfp

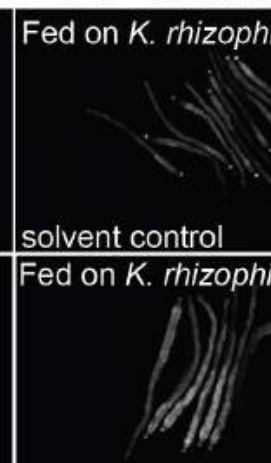

Deat

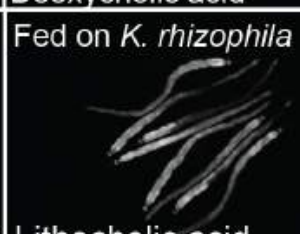

Fed on K. rhizophila

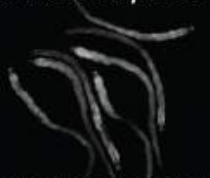

Chenodeoxycholic acid

Fed on K. rhizophila

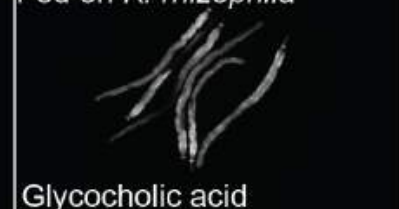

Glycocholic acid

Fed on K. rhizophila

D

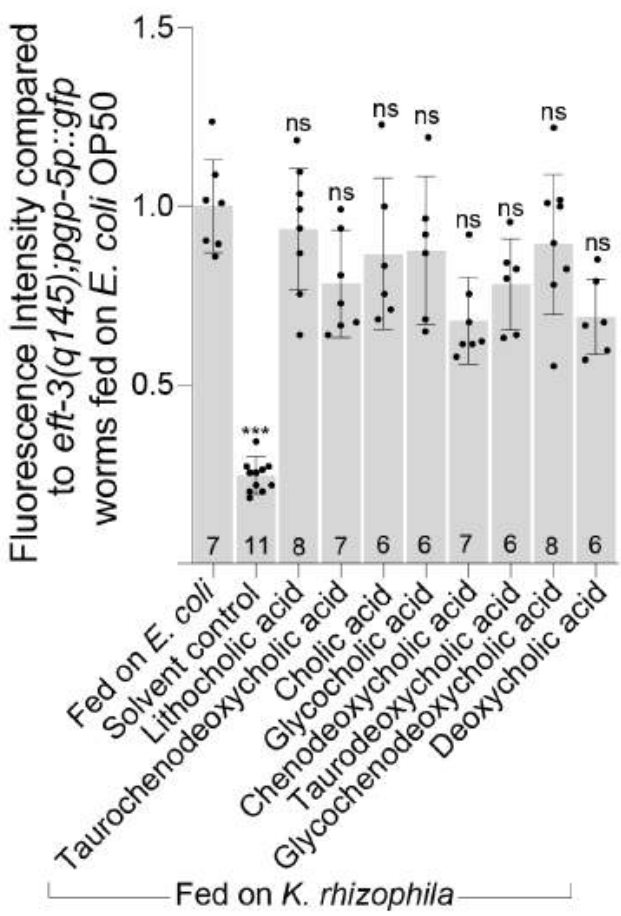


bioRxiv preprint doi: https://doi.org/10.1101/2020.01.08.898668; this version posted January 9, 2020. The copyright holder for this preprint (which was not certified by peer review) is the author/funder, who has granted bioRxiv a license to display the preprint in perpetuity. It is made available under aCC-BY 4.0 International license.

\section{Supplementary Figure 1}

A

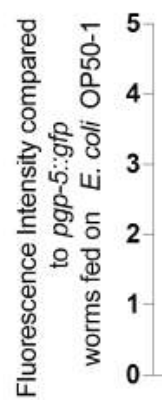

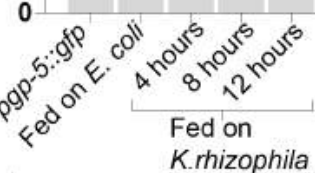

eft-3(q145)

pgp-5::gfp

D

Fed on $K$. rhizophila and 12 hours post-transfer Shifted to $E$. coli OP50-1

Fed on K. mizophila $\begin{array}{r}\text { eft-3(q145);pgp-5p::gfp } \\ p g p-5 p:: g f p\end{array}$
to $p g p-5 p:: g f p$ worms fed on E. coli OP50-1
B

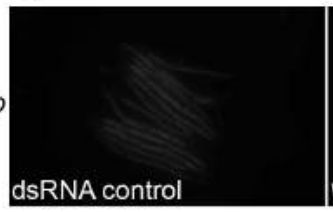

C

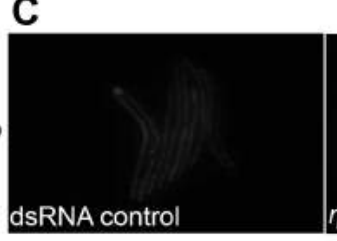

Transferred to

E. coli OP50-1

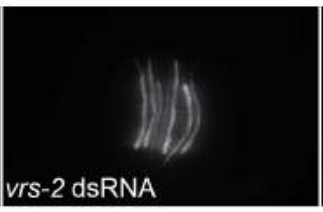

vrs-2 dsRNA

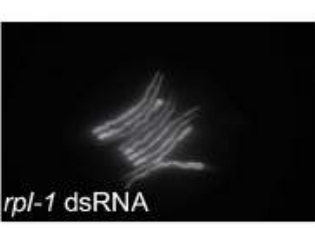

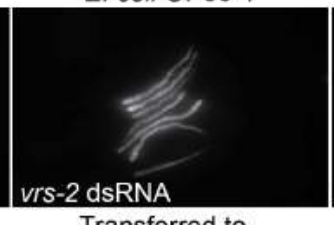

Transferred to

E. coli OP50-1
Transferred to

K.rhizophila

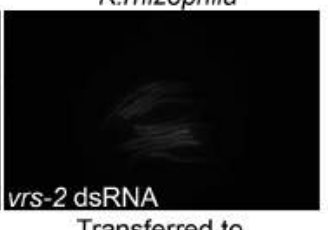

Transferred to

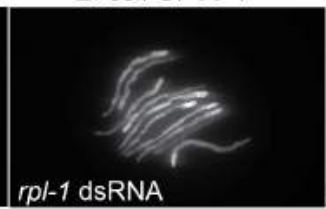

K.rhizophila

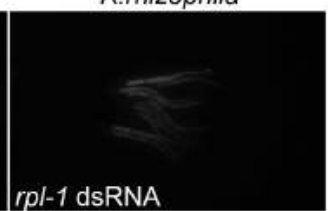

\section{E \\ K. rhizophila $m 6$}

K. rhizophila $m 5$

eft-3(q145); K. rhizophila m4 pgp-5::gfp, K. rhizophila $m 3$

Fed on K. rhizophila m2

K. rhizophila $m 1$ K. rhizophila

E. coli OP50-1

pgp-5::gfp,

K. rhizophila

E. coli OP50-1

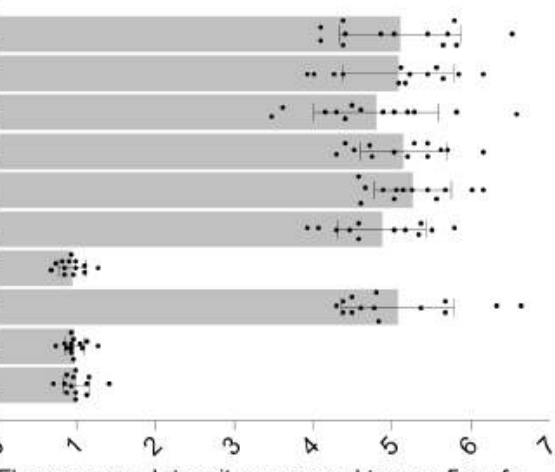

Fluorescence Intensity compared to $p g p-5 p:: g f p$ fed on $E$. coli OP50-1

F

eft-3(q145); pgp-5p:::gfp

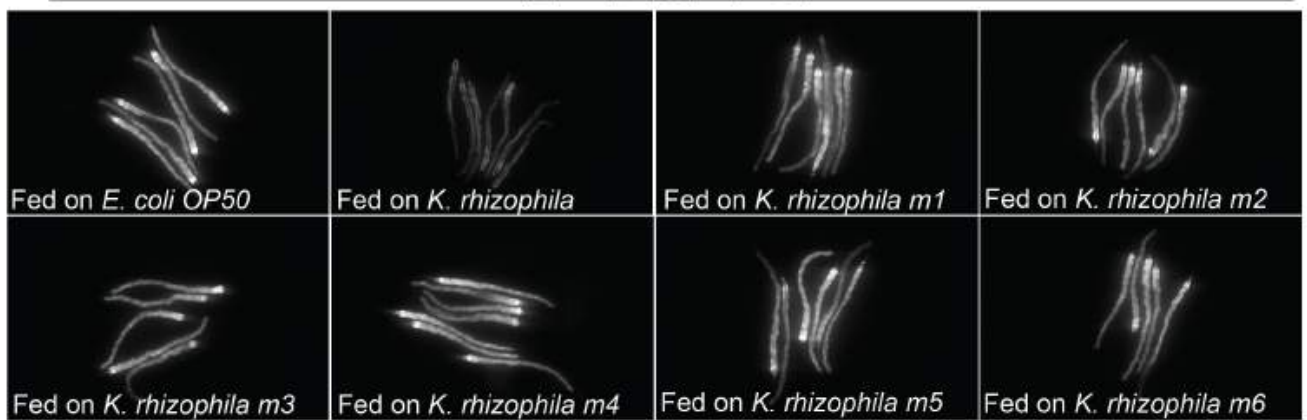


bioRxiv preprint doi: https://doi.org/10.1101/2020.01.08.898668; this version posted January 9, 2020. The copyright holder for this preprint (which was not certified by peer review) is the author/funder, who has granted bioRxiv a license to display the preprint in perpetuity. It is made available under aCC-BY 4.0 International license.

\section{Figure S2}
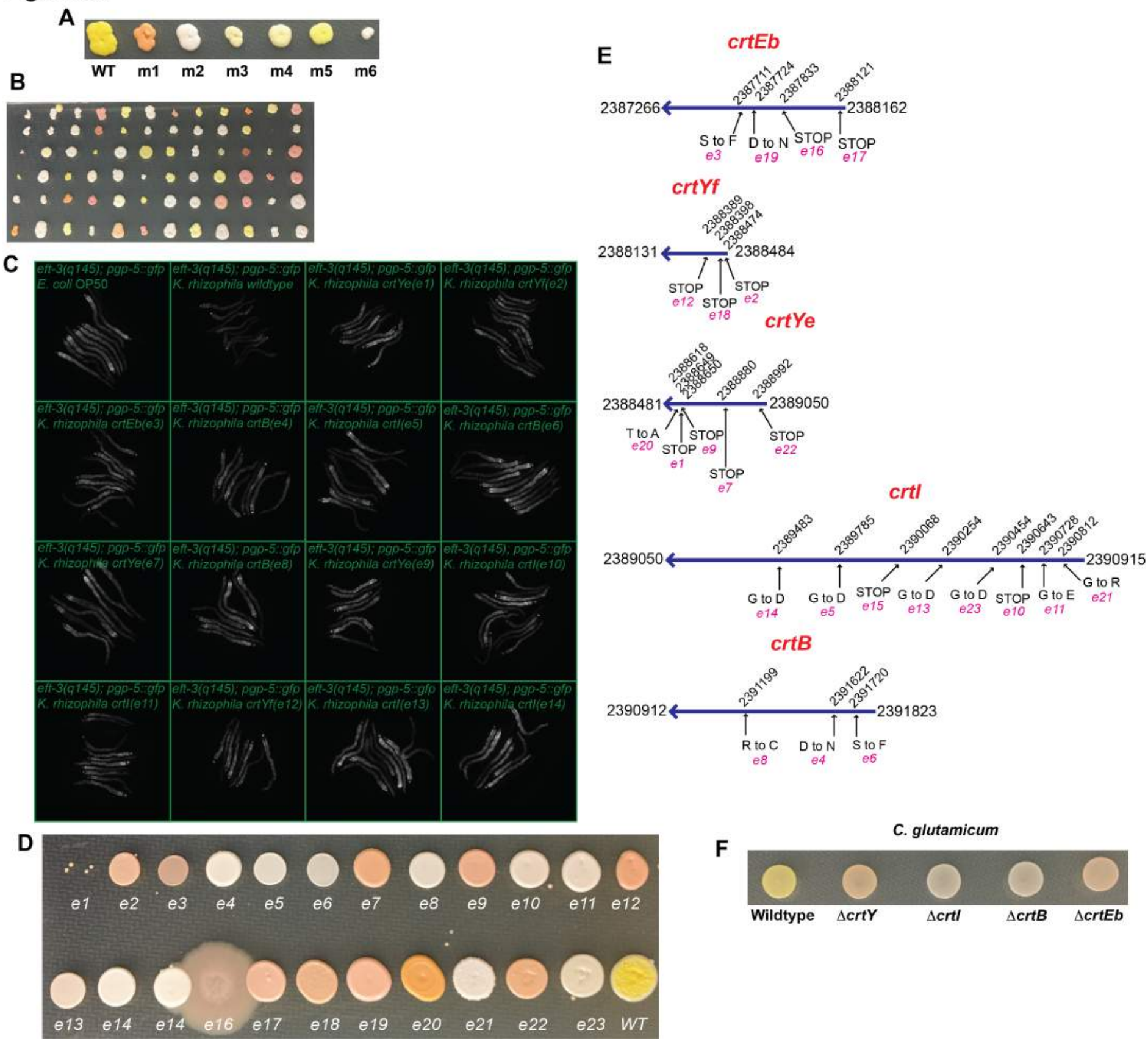


\section{Figure S3}

Arthrobacter Kocuria

Micrococcus Corynebacterium microbacterium leifsonia

Arthrobacter Kocuria Micrococcus Corynebacterium microbacterium leifsonia

Arthrobacter Kocuria Micrococcus Corynebacterium microbacterium leifsonia

Arthrobacter Kocuria Micrococcus Corynebacterium microbacterium leifsonia

Arthrobacter Kocuria Micrococcus Corynebacterium microbacterium leifsonia

Arthrobacter Kocuria

Micrococcus Corynebacterium microbacterium leifsonia

Arthrobacter Kocuria Micrococcus Corynebacterium microbacterium leifsonia

Arthrobacter Kocuria Micrococcus Corynebacterium microbacterium leifsonia

Arthrobacter Kocuria Micrococcus Corynebacterium microbacterium leifsonia

Arthrobacter Kocuria Micrococcus Corynebacterium microbacterium

\section{Crtl Protein Alignment}

\section{R}

1
1
1
1
1 1 MTDGNRTTRHPTRPENAHRGSPDGGAPRTV
$-10-1$
-

E

40
61

44

46

38

38

101 GHLHEPPVDVRT--GHAETLFESLEPGAGRRLRAYLDSASRIYGLAKEHFLYTDFRRPAA

$106--T H D A-V D V P T G R E E A I A L F E S I E P G A G A K L G N Y L D S A A D A Y D I A I D R F L Y N N F S T L G P$

95 PGKGEP-IDIVSGREAVRALF EKHEPGSGDNIDAYLDSAKDAYELSTSKFLYDPYSSTKG

95 DGR-RP-LDIRAA GAANRALF ESVESGAGAALDRYLAGARETYGLAVDRFLYSTEASIRP

\section{D}

154

176

159

163

154

153

214

236

219

223

214

213

264

LLDPR ILRRAPQLAKLLS TSLQS HVARRFAS E IRQVLGY PAVFLGSSPEKTPALYQLMS LARAEVLRNLPRLGGLLSTSM LA HPDVLRALPAL G P OLL GGLRS HVAARF ODPRLRO ILGY PAVF LGTS PDRA PAMY HLMS LLH RDVLTRAGRLF SLLTRSL $Q K Y$ VN S QF S SPVLRQ I LTY P AVF LSSRPTT TPS MY H LMS LRDPALVKRLPTL I PLLTRTLWKRVT S DFKNTRLOQ I LAY P AVF LGGSPFEVPS L Y HLMS LLS REVLARTGRLARLLLE PLDRYAARCVRDTVLRQI LGY PAVF LGTS PDRAPS LYHLMS

HLDLADGVKY PMGGF AAVADAMA QLARK HGAOT $Q L$ GATATA I ETS TGKNL------HLDLTDGVQY PVGGF AALVDAMERLVRAAGVE IVTGAEVTGI EVAPAPASLRSRVGAA-HLDLADGVQY PLGGF AALVDAMAEVVREAGVE IRTGVEATAVEVADRPAPAGR - LGRLAA HTDLVQGVKY P I GGFTAVVNALH QLALENGVEFOLDSEVISINTASSRGHLDLGDGVLY PKG GMTE I ITAIE KLARGRGVTIETSAPVEA I ITE SGTA HLDLDDGVRY PVGGFATIIDRIVAFARAAGAEIVIDARVTGIRTGVGGR - . . . .

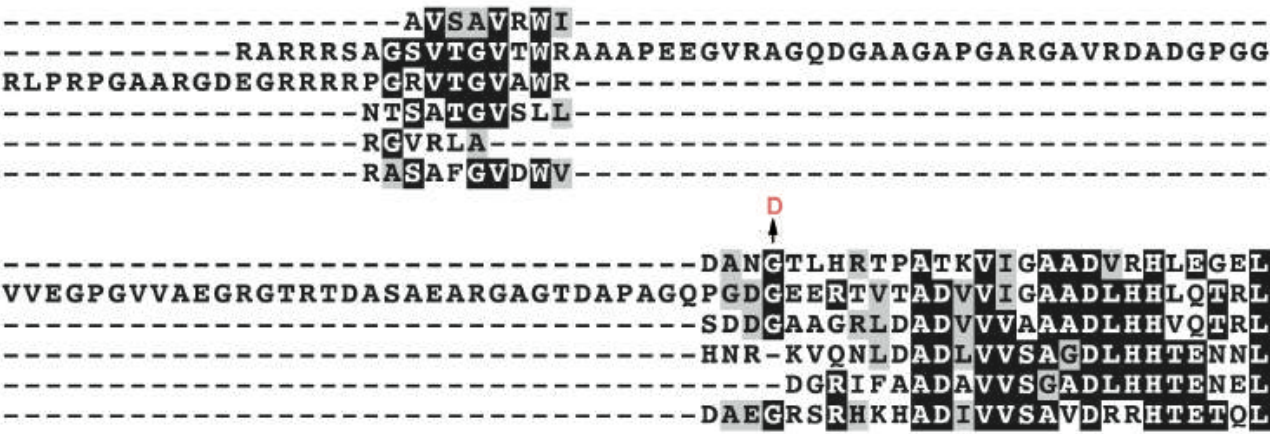

LPESLQTH TAKSFARKDPGPSAVLLCLGIKGKLPQLEHHNLLFTEDWSENFSRTROGRE LPD DFRAP -ES RWTHRD P GPS GVLVCL GVRGKLPQLVHHNLLF TADWDDNF GR IADGTPLPPGRRVA-ESTWDRRD P GPS GVLVCVGVRGSLPQLAH HTLLF TADWEDNF GR IERGEDLPRELRTY PER YWSNRNPGI GAVLI LLGVKGELPQLDHHNLFF SEDWTDDFAVVFDGPQL LEEKDRQY PEKWWKDKVPSPGALLLLLGVTGELPQLTHHTLLFTDDWHTNFDAIFGENK LPPALR-S S DREWKRRDPGPGAVLAMLGVRGELPQLTH HNLFFTTDWEANFERVFGADR-

D

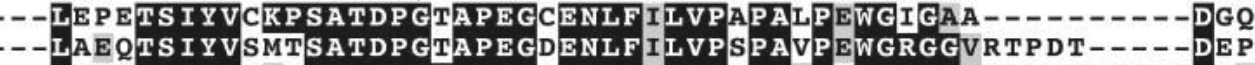
- - LAADTS I Y VSRTSATDPGVAPEGDENLF ILVPAPAEP GWGRGGIRVRDGQGWRVDRA TR P HNASNS I Y VSTRSTSEDGVAPAGYENLFVLIPTKASSS IGHGDAY - - - - - -MQS $--K$ I P D P AS I Y I CRP SASDDSVA P EGHENLFVLVPVPADPDS GRGGVS-- - - - - G-A - GVPDPASIYVCK P SATDPGVAPPGHENLFVLVPVPADTS IGSGGID - . - . - -G-G

GDAAVEAVAEAA IDQLS AWARTDDLRER IVVRQSIGPGDFA $Q Y$ GAYRGGALGLAHTLGQ GSPQVERVADAA I AQLARWAEI P DLAER I VVRRTYGP GDFEAQF NAWR GSML GP GH TLRQ GDAOVEAVADRALDQLARWAG I P DLAER I VVRRTY GPGDF AADVHAWRGSLLGPGHTLA ASASVET IASHA INQIATQAG I P DLTDR I VVRRTIGPADFE HRYHSWVGSALGPAHTLRQ GDER IEKAADRVIAQI GEWTG I P DLAER I VVRKT IAPEDFK DLHAWHGN SLGLAHTLNQ 
bioRxiv preprint doi: https://doi.org/10.1101/2020.01.08.898668; this version posted January 9, 2020. The copyright holder for this preprint (which was not certified by peer review) is the author/funder, who has granted bioRxiv a license to display the preprint in perpetuity. It is made available under aCC-BY 4.0 International license.

Figure S4

\section{CrtB Protein Alignment}

\author{
Corynebacterium \\ Microbacterium \\ Leifsonia \\ Arthrobacter \\ Kocuria \\ Micrococcus \\ Corynebacterium \\ Microbacterium \\ Leifsonia \\ Arthrobacter \\ Kocuria \\ Micrococcus \\ Corynebacterium \\ Microbacterium \\ Leifsonia \\ Arthrobacter \\ Kocuria \\ Micrococcus
}

Corynebacterium Microbacterium

Leifsonia

Arthrobacter

Kocuria

Micrococcus

Corynebacterium Microbacterium

Leifsonia

Arthrobacter

Kocuria

Micrococcus

Corynebacterium Microbacterium

Leifsonia

Arthrobacter

Kocuria

Micrococcus

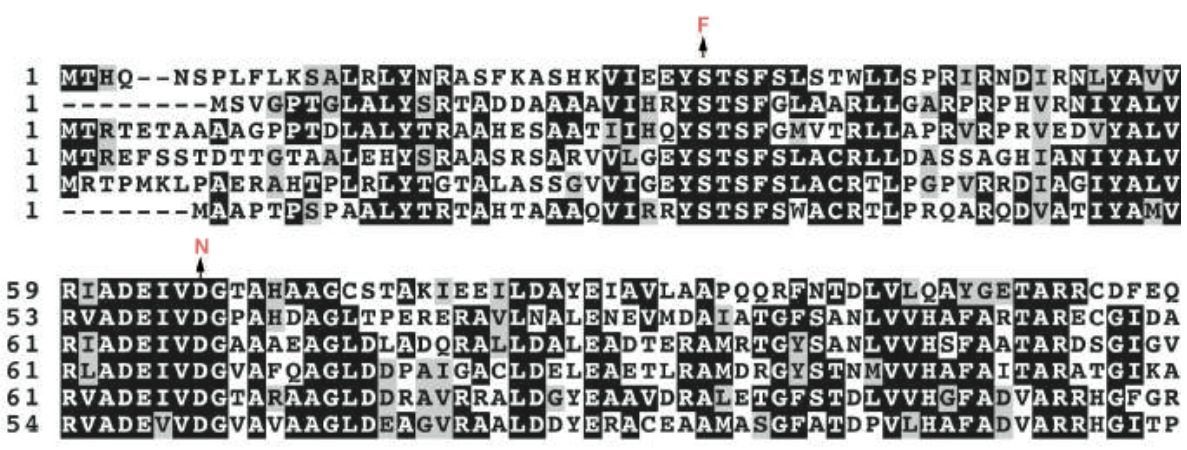

119 EHVIAFFASMRKDLKANTHDPDSFTTYVYGSAEVIGLLCLSVFNOGRT- - ISKKRLE IM

113 DLIAPFFASMRTDIDTAEHDDLSHDAYVYGSAEVVGLMCLOVF LNAGMSAPAR - - PAADI

121 ALTRPFFASMRRDLSLVDFTADELREYVYGSAEVVGLMCLAVFLTDSP---VADDRRRRL

121 ELTTPFFASMRADLSTGEHDARSLQEY IYGSAEVIGLMCLQVFAAMPGAPQLNRAEEQRT

121 ELTEPFFASMRADLEVAEHDGASLEDY Y GSAEVVGLMCLEVFTDMPGTRAQTPEOREMI

114 ELTRPFFASMRADLGIREHGAESLDAYI HGS AEVVGLMCLQVFLSLPGTRART PGORQEL c

176 QNGARSLGAAFOKINFLRDLAEDQONLGRF YFEKTSOGTLTKEOKEDLIADTRQDLAIAH 171 VDGARRLGAAF QDVNFLRDLADDADRLGRDYLDGAA----DDDRRTAVLDRI DADLAAAA 178 EAGARRLGAAF OKINFLRDLAADY AGLGRSYFPGIDPARLTEROKLALVVDI DGDLGAAA 181 KLAARSLGAAFOKVNFLRDLAODS QELGRTYF PGMDPEGFDEQGKALLVAQINODLAAAR 181 RSTARRLGAAFQKVNFLRDLGADHDQLGRTYLPGADPAHLTEDRKAALLADLDADLDAAV 174 RAQASRLGAAFQKVNFLRDLAADH HELGRTYLPGAAPGVLTEARKAELVAEVRADLDAAL

236 DAFPEIPVQARI GVI SAYLLFQKLTDR I EATPTADLLRERI RVPL H IKLSTLARATMKGL 227 SVIPHLPPDCRAAVT AAHDLFAELSRRLRLSPAGA---PRVRVPDGVKATLAARALLGRP 238 DAIAELPGNCRRAIVAAHALF SELSDR IRATPARDLIVRRVSVPMRTKLAILLRATAGIL 241 AGLPYLAPOAARAVCLAHDLF QELNVQLEKVPAAALLRTRISVSAPRKAMI ALRVIIGAG 241 PGILALDRRARRAVS MAHGLFTELARRIERVPARELSTRRVSVPTAVKLOIAARAIIADTE 234 PGIRVLDGGAGRAVALAHGLFAALVDRIEATPAAELAHRRVRVPDHOKARIAARVLARGR

296 SMSIYRKNS---- -

284 PKGPRP-------

298 R-D.--

301 TPSHHKLRMEVSSR

301 VTA---.-----

294 RGGRR--- 
bioRxiv preprint doi: https://doi.org/10.1101/2020.01.08.898668; this version posted January 9, 2020. The copyright holder for this preprint (which was not certified by peer review) is the author/funder, who has granted bioRxiv a license to display the preprint in perpetuity. It is made available under aCC-BY 4.0 International license.

\section{Figure S5}

\section{CrtEb Protein Alignment}

\author{
Corynebacterium \\ Arthrobacter \\ Kocuria \\ Micrococcus \\ Microbacterium \\ Clavibacter \\ Leifsonia \\ Corynebacterium \\ Arthrobacter \\ Kocuria \\ Micrococcus \\ Microbacterium \\ Clavibacter \\ Leifsonia
}

Corynebacterium

Arthrobacter

Kocuria

Micrococcus

Microbacterium

Clavibacter

Leifsonia

Corynebacterium

Arthrobacter

Kocuria

Micrococcus

Microbacterium

Clavibacter

Leifsonia

Corynebacterium

Arthrobacter

Kocuria

Micrococcus

Microbacterium

Clavibacter

Leifsonia

Corynebacterium

Arthrobacter

Kocuria

Micrococcus

Microbacterium

Clavibacter

Leifsonia

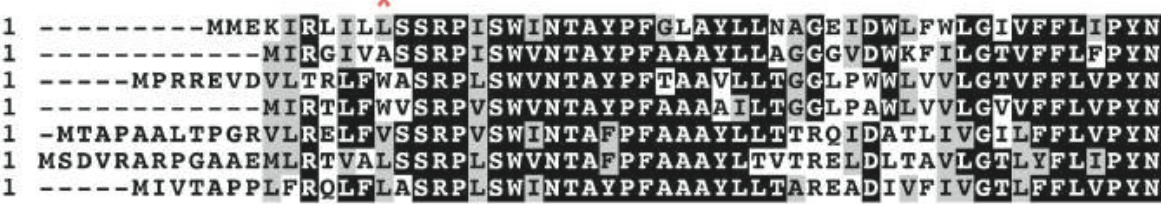

52 IAMYG INDVFDYESDMRNPRKGGVEGAVLPKSS-HSTLLWASA IST IPFLVI-- - -LF IF 49 LLMYGVNDVF DY ES DMRNPRKGGIEGAVLSKOS - HKALL IACTVCS LPFLIV - - - LAAG 56 LAMY G INDVFDYESDLRNPRKGGVEGAVVDRAA-ORPVLLASCLVPAPFVLVLGGYAVVT 49 LAMYGINDVFDFASDLRNPRKGGVEGSVLGDPAVRRRVLAWSVLLPVPFVAVLAGWSAVR 49 LAMY G INDVF DFASDLRNPRKGGVEGSVLGDPAVRRRVLAWSVLLPVPFVAVLAGWSAVR
60 LAMY GVNDVFDYESDLRNPRKGGTHGAVLDKRM-BPI TLWASVLSCLPFVVY--- -LVVV 61 LAMY G INDVFDYESDMRNPRKGGVEGAVLARAM-HRPVLLAVLVTNVPFLVY - - - LVIV 56 LTMYGVNDVF DYASDLRNPRKGGVEGALLDPGT-BRRTLVAAAATSLPFLVF--- - LALA

107 GTWMSSLWLTLSVLAVIAYSAPKLRFKERPFIDALTSSTHFTSPALIGATITGTSPSAAM 104 GDAASN ITLAVSIFAVIAY SAPRLRFKERPGIDSITSAVHFVSPAVYGWVLAGSAVOAEO 115 GNWVSI AVLAVSLF AVVAY SWAGLRF KERPFVDAMTSATHFVSPAVY GLTLAGATFTPGL 109 GEWAAVLVLAVSLF AVVAY SWAGLRF KERPF LDAATSATHFVSPAVYGLALAGATPTPAL 115 GS PLSWLVLALSLFFVVFY SAP PLRLKERPFADSVTSSIHFFSPAVYGLVLAGAVWTWOL 116 GSAASI AVLAVSVFAVIAYSLKGLRFKERPVLDSLTSSTHFTSPAVYGIVLAGGAFTPAL 111 GPPLSWAVLAGSLFFVLAYSVRGLRFKKVPFLDSATSSIHFVSPALYGLVLAGATFTPGL

167 WI ALGSFFLWGMASQ I LGAVQDVNADREANLSS I ATVIGARGA IRLS VVLYLLAAVLV-T 164 WMVF LAFLLWGMAS HALGAIODI IPDROGGL GS I TVLSARKTI YLVLACYLLAGGLVAV 175 WALLI GFFLWGMA SQMFGAVQDVVPDREGGLASVATVLGARPTVWVAALLYALAGGLM-V 169 AALL GAFFLWGMA SQMF GAVQDVVPDREGGLASVATVLGARRTVLLAAGLYAAAGLLL-L 175 VFVFVAFALWGIA SAF GAVQDVEADRAADISS IATARGARWTVRFALVAYALAGVAM-L 176 WA ILAAFFLWGVASHAFGAVODIVADREGGISS I TVLGGAVTVRIAVLAYAAAGVAM-I 171 WLVLFAFFLWGVGSHAFGAVQDVAPDREAGIASVATVLGAARTVRFAIAAWALAALAA-L

226 TLPNPAW I IGI A I T TVFNAARFWNITDASCEQANRSWKVFLWLNYFVGAVITILLIA I H 224 GVSGIGRWAAVISLPYVLNVLPHLGISDASSGTVNRGWKRF LWINYLCGFLLTMLLIF SA 234 FTEWPGQLAALLA VP Y LLNVLRFS GVTDT DSGRANAGWKTF LWLNYLT TF LVTMLL IWWA 228 ATDPPGPLAALLA VP Y V VNLRFRRITDATSGAAHRGWOLF LPLNY TTGF LVTLLL I GWA 234 FTAWPGPLAGVLVIPYLVVCWPYRNVTDAESDRATAGWNRF LWLNQI AGFGTTMLLIWWW 235 FTGLPGI IAAVLV IPY I LSTAPFWSIRDEDAGAANRGWRRF LGLNF L S GFVVTMLL IAYW 230 A I PWPGPLVAMLALPYITVAAPFWSVPDDRASAANRGWLRFLGTNYACGCLLTLLLIGYA 
bioRxiv preprint doi: https://doi.org/10.1101/2020.01.08.898668; this version posted January 9, 2020. The copyright holder for this preprint (which was not certified by peer review) is the author/funder, who has granted bioRxiv a license to display the preprint in perpetuity. It is made available under aCC-BY 4.0 International license.

\section{Figure S6}

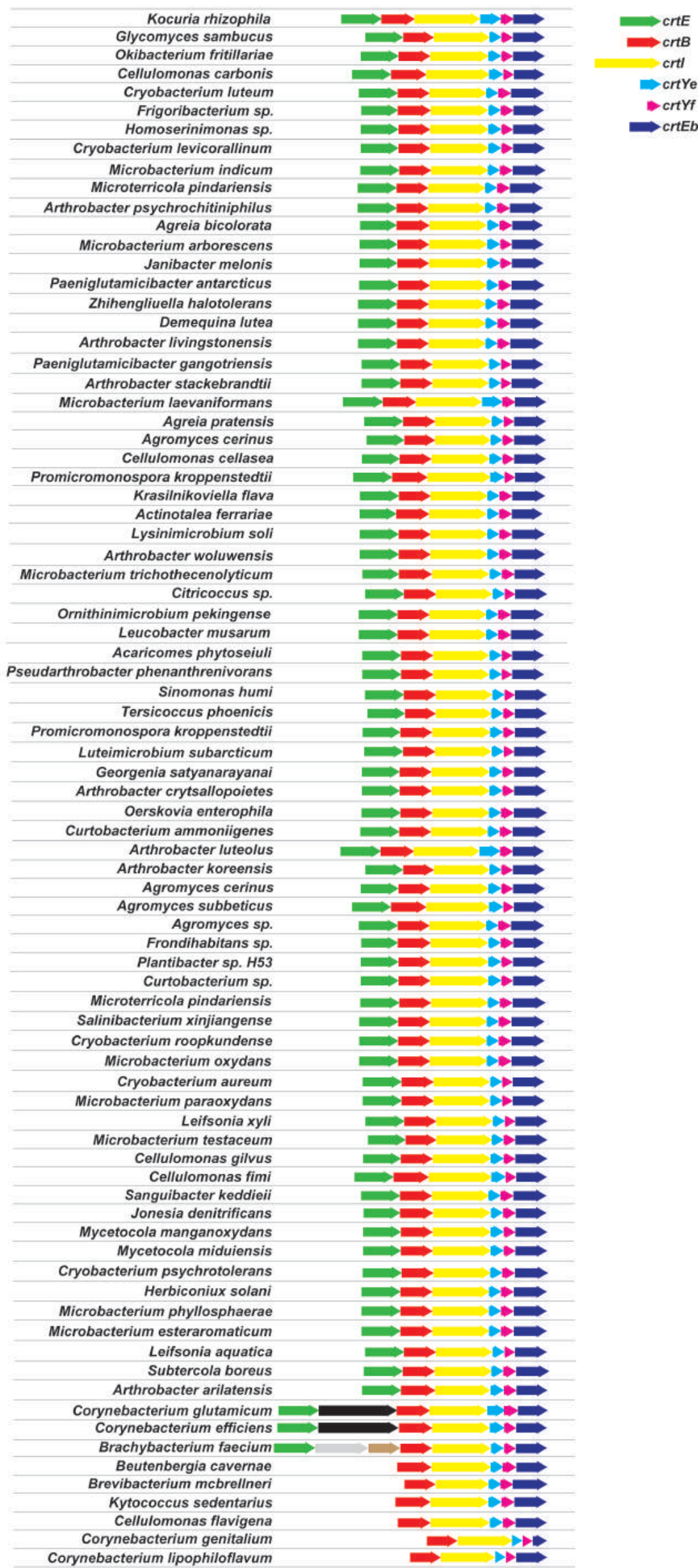


bioRxiv preprint doi: https://doi.org/10.1101/2020.01.08.898668; this version posted January 9,2020 . The copyright holder for this preprint (which was not certified by peer review) is the author/funder, who has granted bioRxiv a license to display the preprint in perpetuity. It is made available under aCC-BY 4.0 International license.

\section{Figure S7}

A

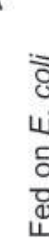$$
\text { A }
$$

pgp-5p::gfp

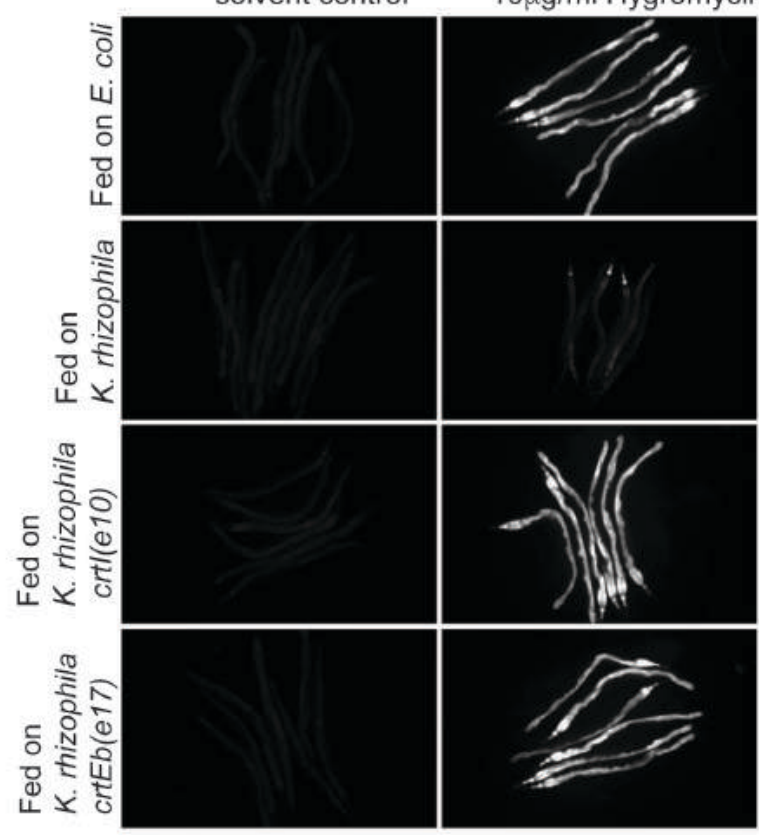

D

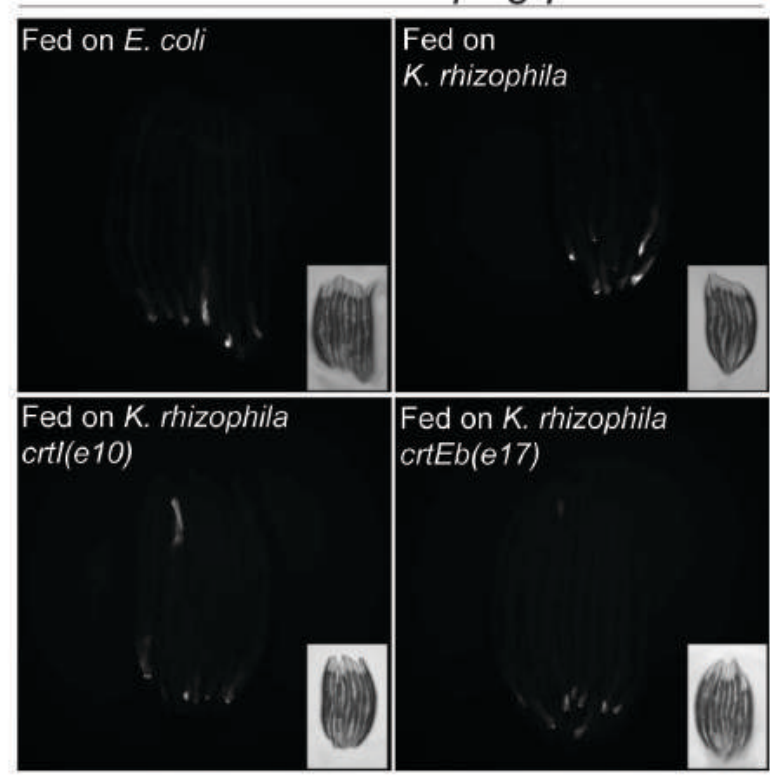

B

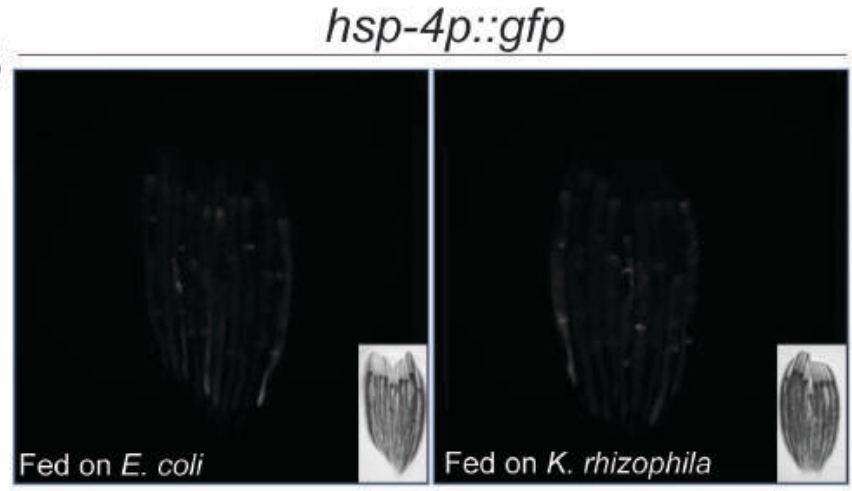

C

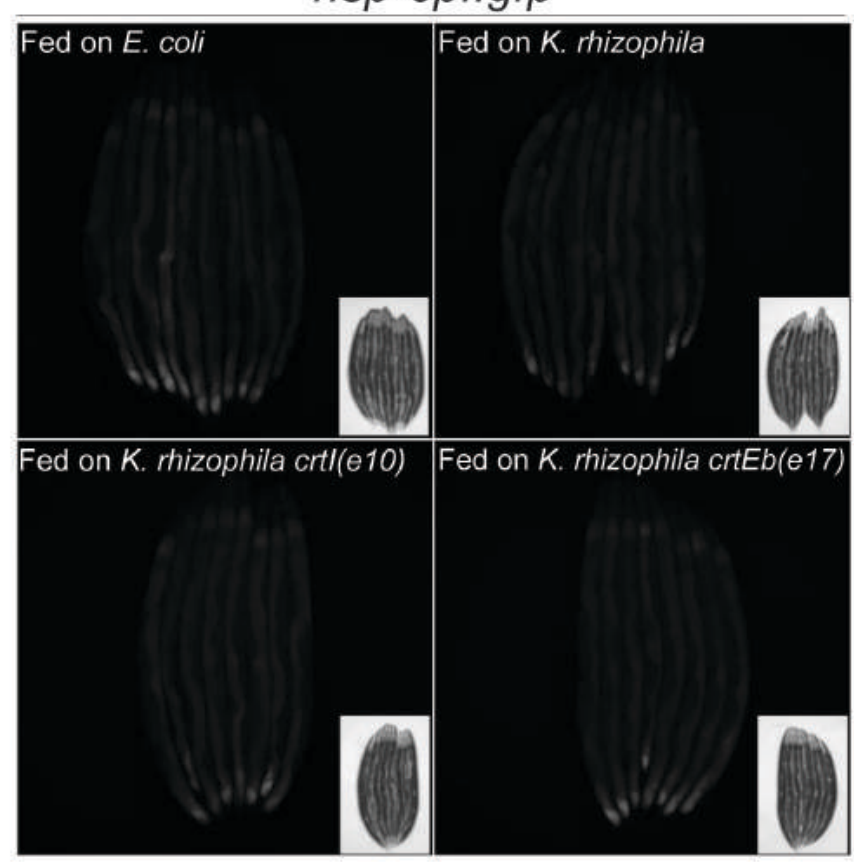

E

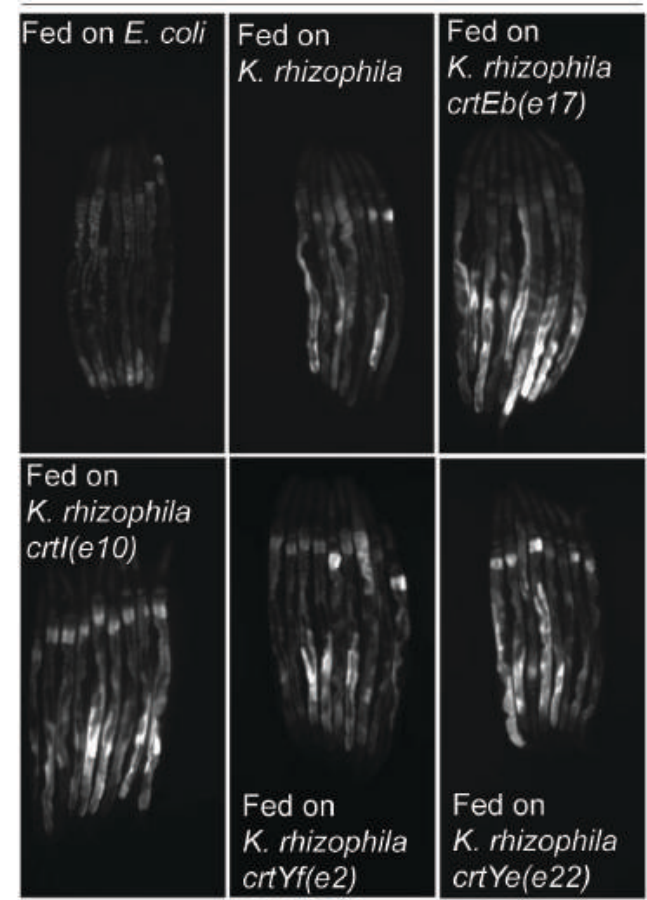


bioRxiv preprint doi: https://doi.org/10.1101/2020.01.08.898668; this version posted January 9, 2020. The copyright holder for this preprint (which was not certified by peer review) is the author/funder, who has granted bioRxiv a license to display the preprint in perpetuity. It is made available under aCC-BY 4.0 International license.

\section{Extraction of Kocuria Carotenoids}

Grow Kocuria in LB media overnight at $37^{\circ} \mathrm{C}$

Centrifuge at 4000 RPM for 15 min

Wash with water

Wash with Acetone

Extract in methanol at $60^{\circ} \mathrm{C}$ (Multiple rounds)

Filter

Add 2 volumes of $15 \% \mathrm{NaCl}$

Mix and add hexane (multiple-rounds)

Remove hexane layer and wash with water Atleast three times

Evaporate or speed vac to concentrate

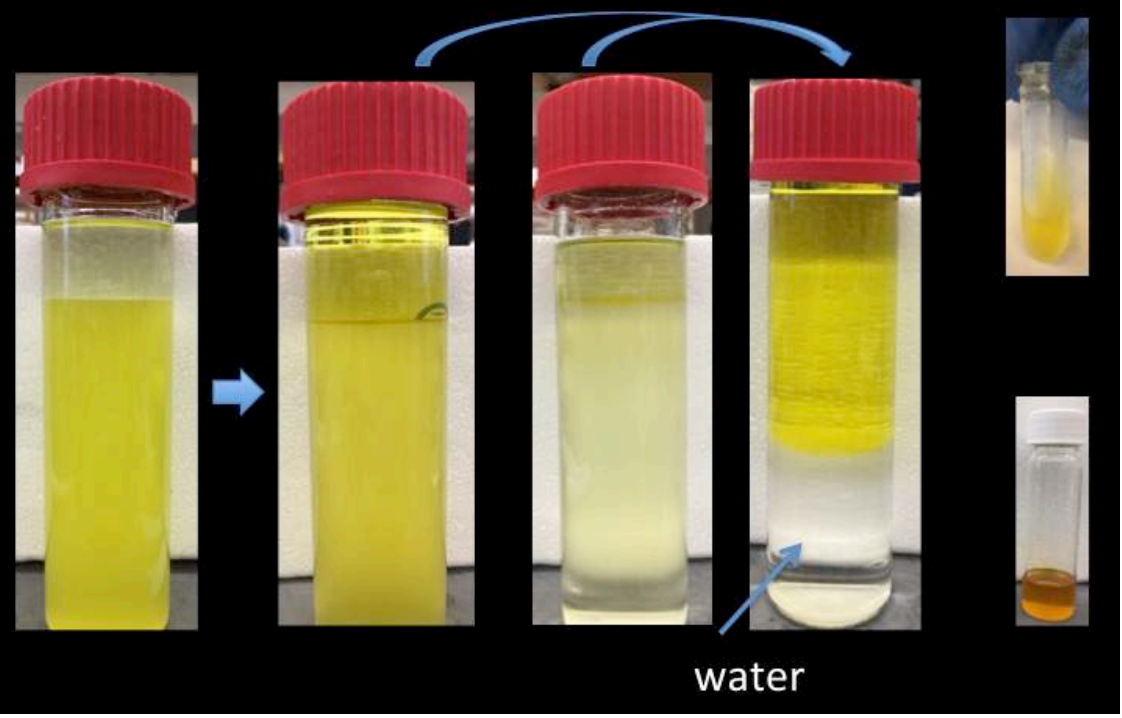


bioRxiv preprint doi: https://doi.org/10.1101/2020.01.08.898668; this version posted January 9,2020 . The copyright holder for this preprint (which was not certified by peer review) is the author/funder, who has granted bioRxiv a license to display the preprint in perpetuity. It is made available under aCC-BY 4.0 International license.
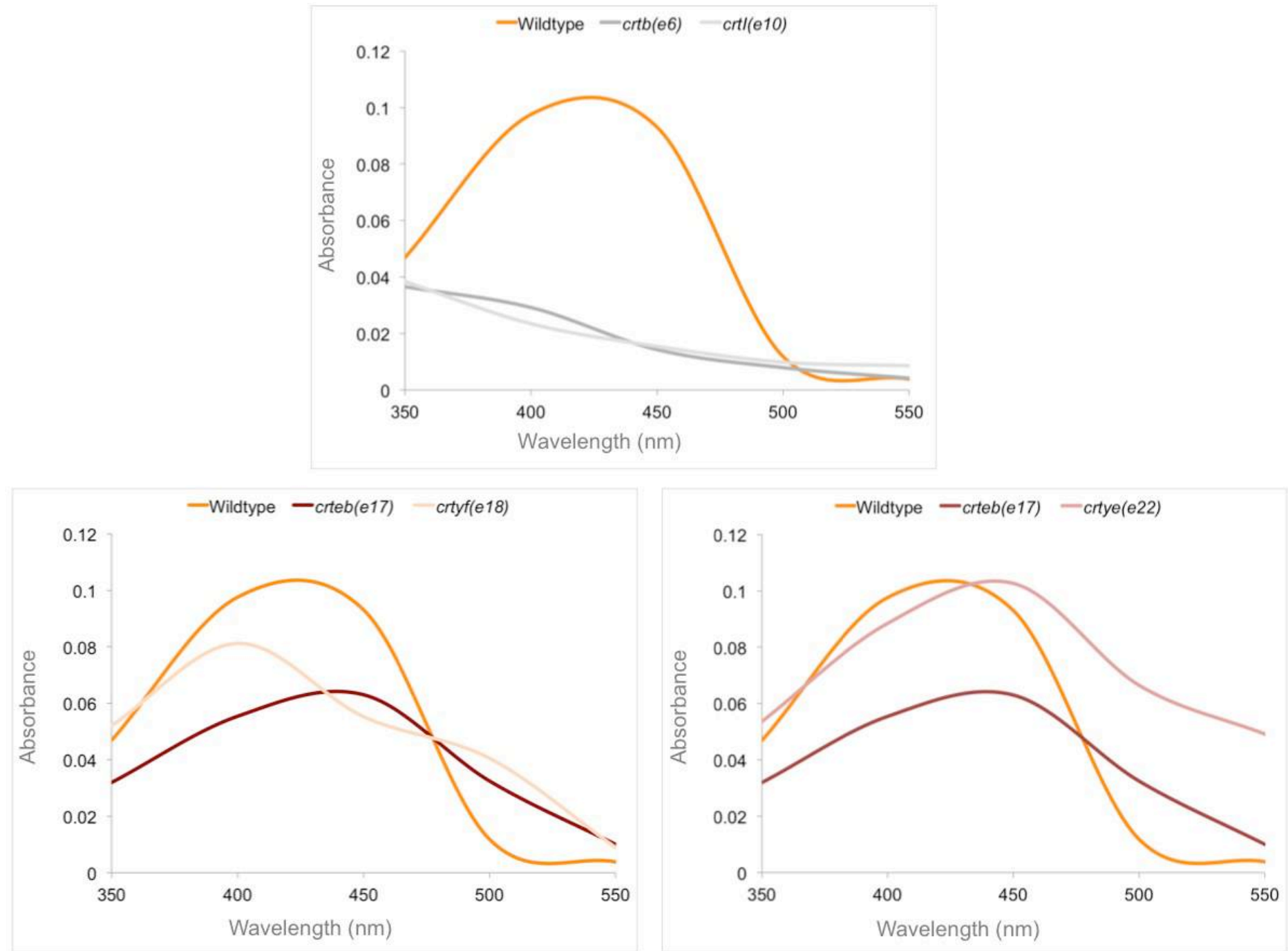
bioRxiv preprint doi: https://doi.org/10.1101/2020.01.08.898668; this version posted January 9,2020 . The copyright holder for this preprint (which was not certified by peer review) is the author/funder, who has granted bioRxiv a license to display the preprint in perpetuity. It is made available under aCC-BY 4.0 International license.

\section{Figure S10}

A

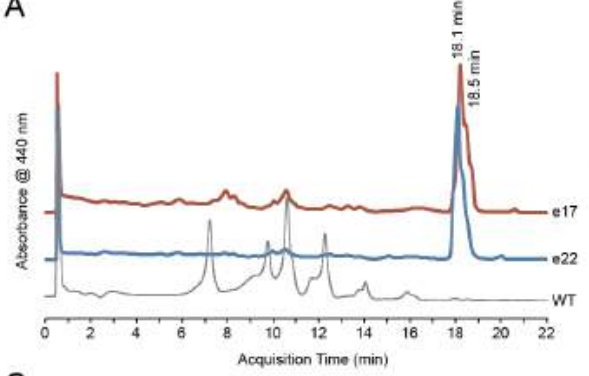

C

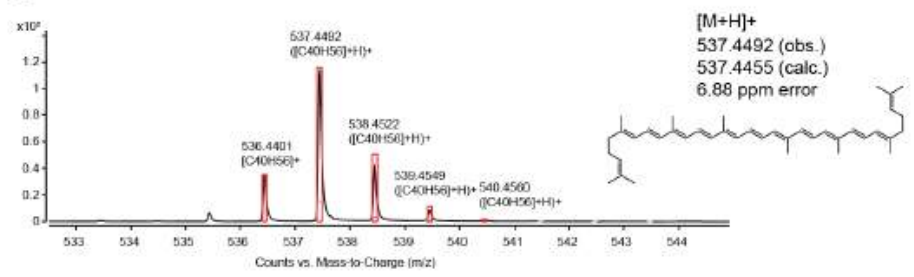

B

537.4492 (obs. $6.88 \mathrm{ppm}$ error

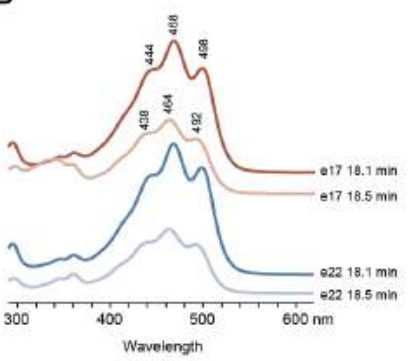


bioRxiv preprint doi: https://doi.org/10.1101/2020.01.08.898668; this version posted January 9,2020 . The copyright holder for this preprint (which was not certified by peer review) is the author/funder, who has granted bioRxiv a license to display the preprint in perpetuity. It is made available under aCC-BY 4.0 International license.

\section{Figure S11}

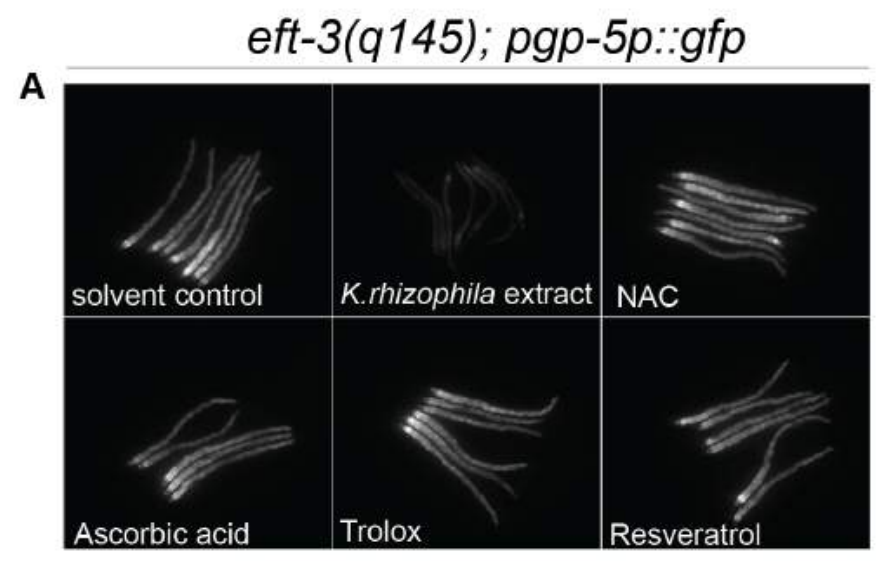

B

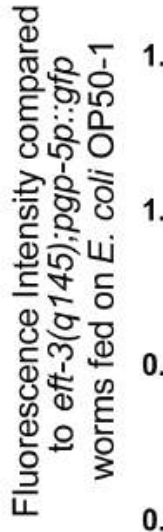

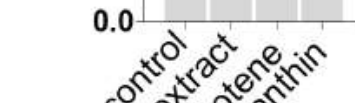

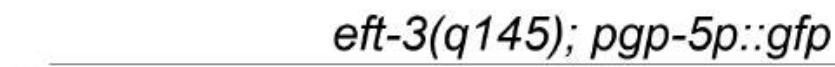

C

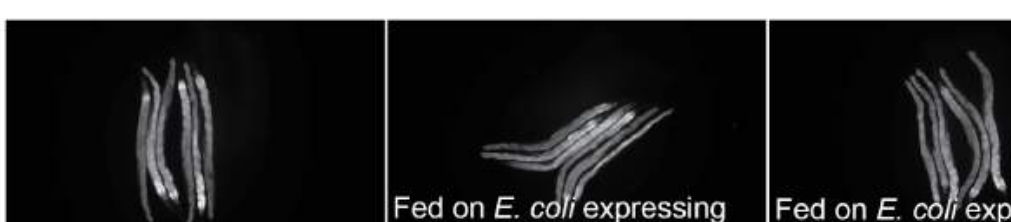

Fed on Control E. coli neurosporenone

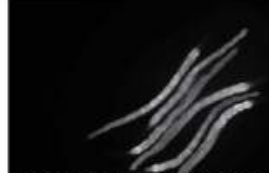

Fed on $E$. coli expressing zeaxanthin

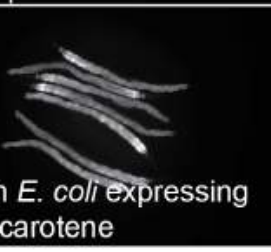

Fed on E. coll
alpha-carotene
Fed on $E$. coli expressing Violaxanthin

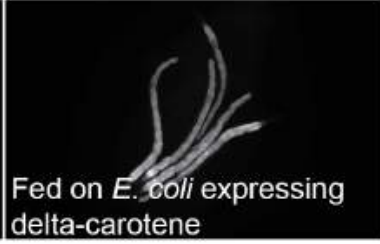

$10 \mu \mathrm{g} / \mathrm{ml}$ Hygromycin

\section{pgp-5p::gfp}

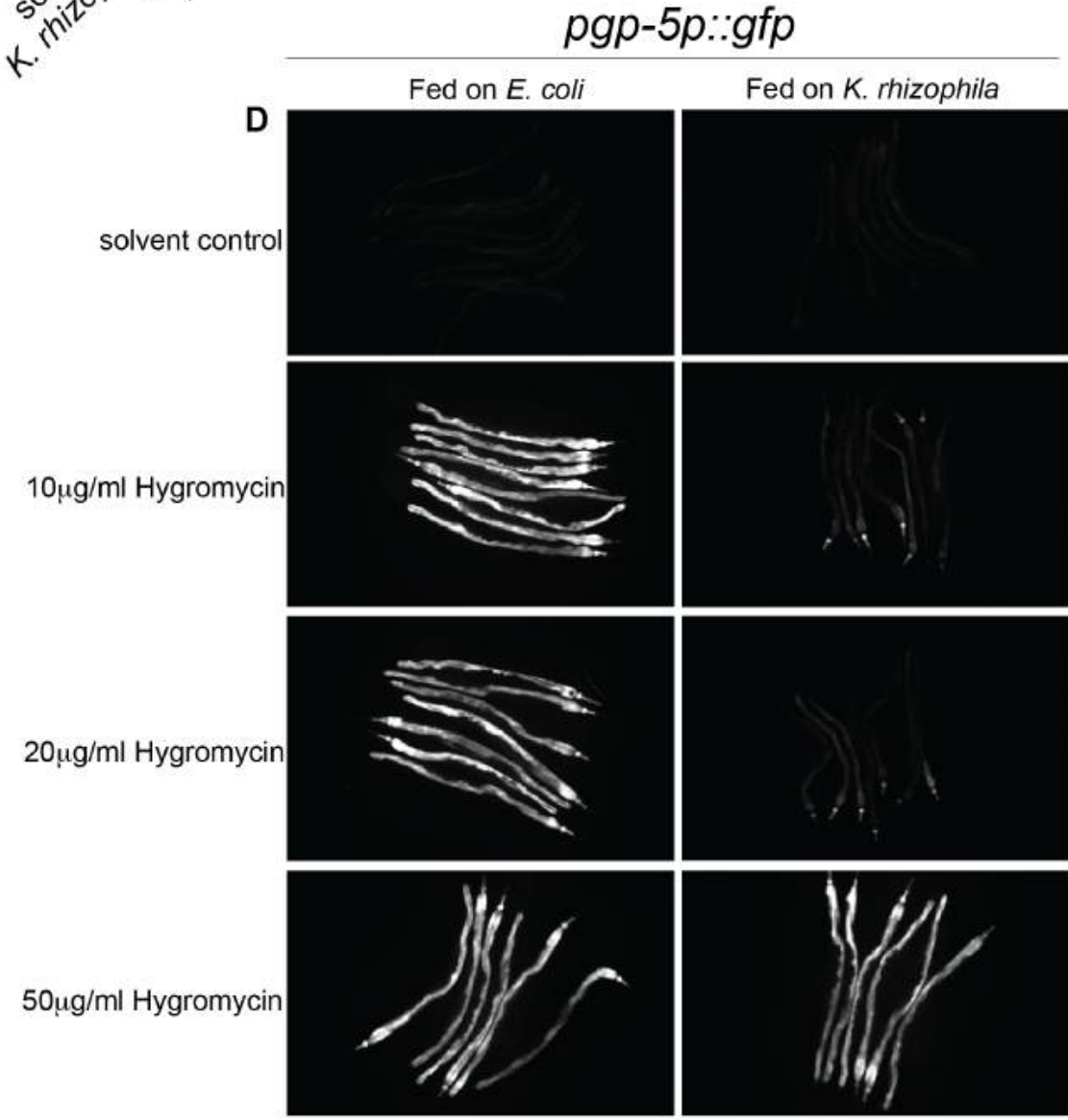


bioRxiv preprint doi: https://doi.org/10.1101/2020.01.08.898668; this version posted January 9,2020 . The copyright holder for this preprint

(which was not certified by peer review) is the author/funder, who has granted bioRxiv a license to display the preprint in perpetuity. It is made available under aCC-BY 4.0 International license.

\section{Figure S12}

A
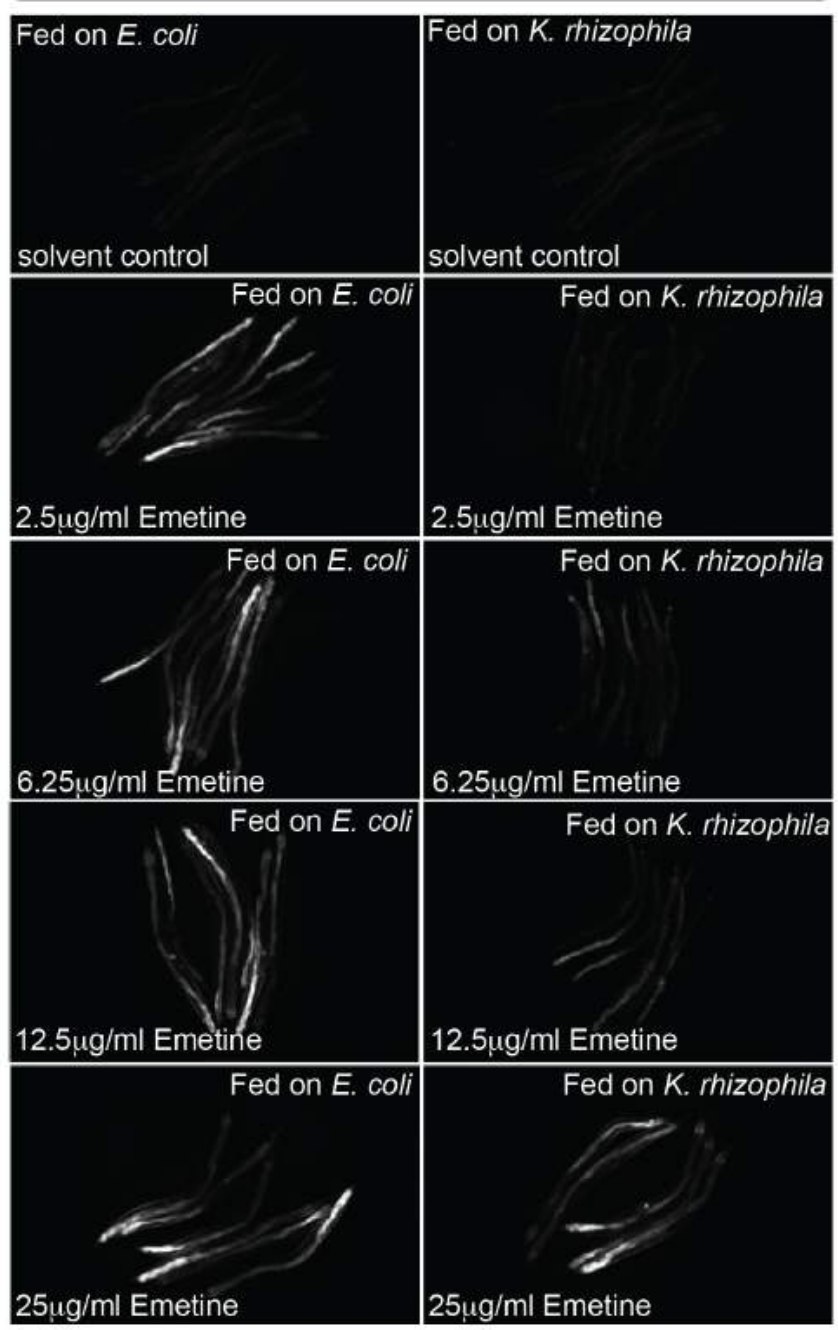

c

$$
\text { pgp-5p::gfp }
$$

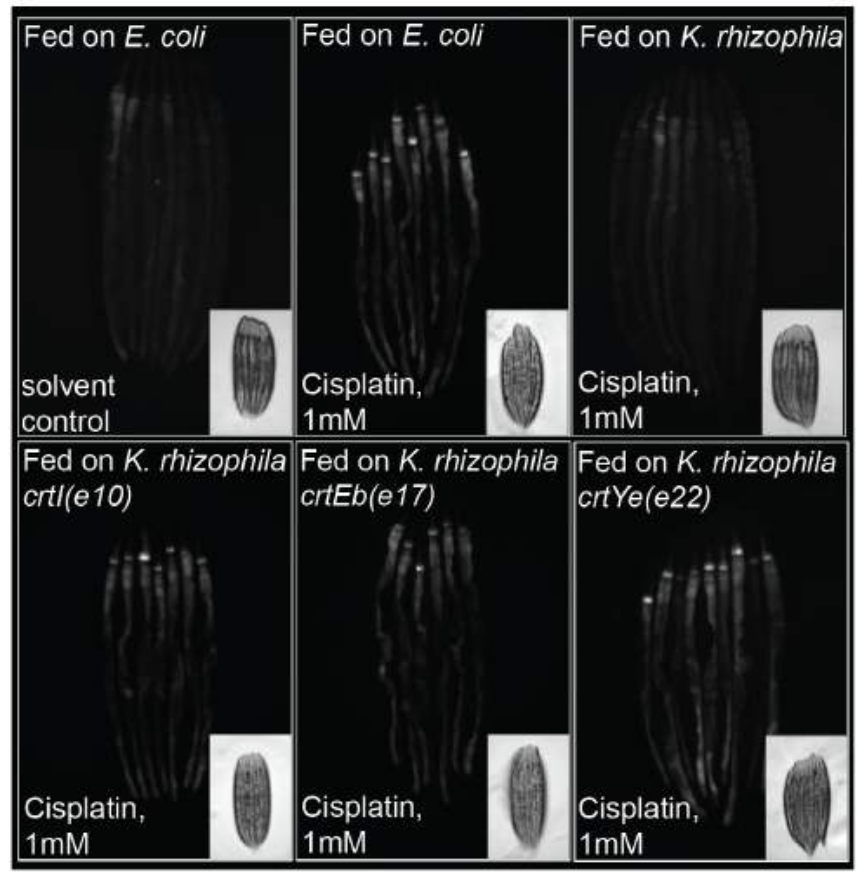

B

$$
\text { pgp-5p::gfp }
$$

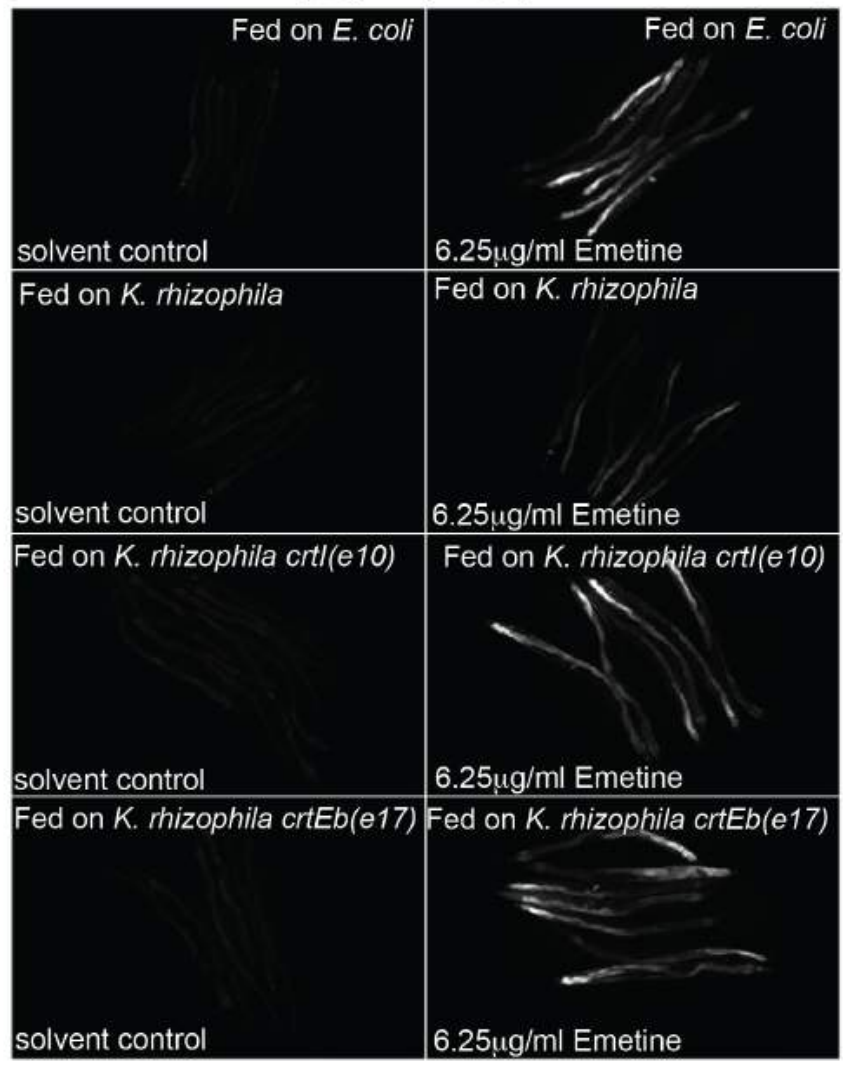

D Solvent

둥휴
के 융

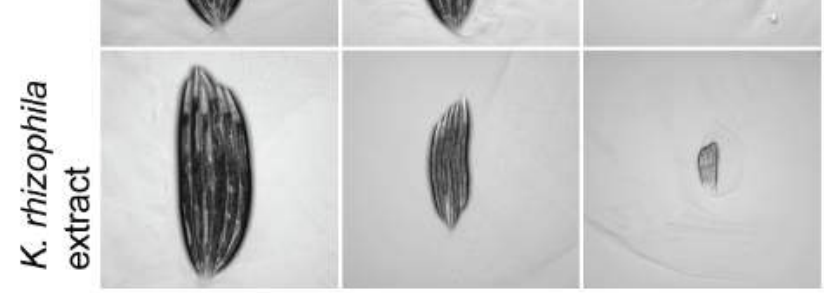

E

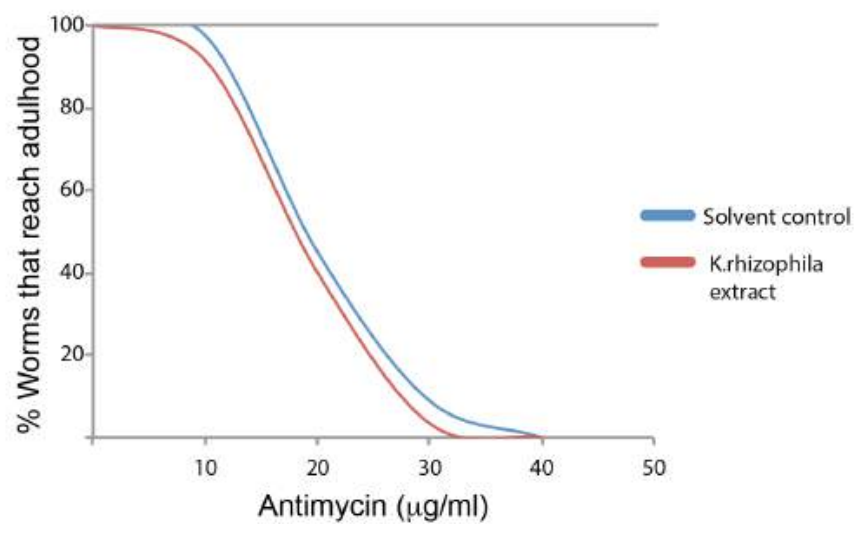


Supplementary Table 1

\begin{tabular}{|c|c|c|c|c|}
\hline \multirow[t]{2}{*}{$\begin{array}{l}\text { Kocuria } \\
\text { genotype }\end{array}$} & \multicolumn{2}{|c|}{$\begin{array}{c}\% \text { eft-3(q145); pgp- } \\
\text { 5::gfp animals showing } \\
\text { GFP induction }\end{array}$} & \multicolumn{2}{|c|}{$\begin{array}{c}\% \text { pgp-5::gfp animals } \\
\text { showing GFP } \\
\text { induction }\end{array}$} \\
\hline & $\%$ & $\mathbf{N}$ & $\%$ & $\mathbf{N}$ \\
\hline Wildtype & 0 & 26 & 0 & 24 \\
\hline crtYe(e1) & 95.6 & 23 & 0 & 24 \\
\hline $\operatorname{crtYf}(e 2)$ & 94.4 & 36 & 0 & 23 \\
\hline crtEb(e3) & 76.6 & 30 & 0 & 24 \\
\hline $\operatorname{crtB}(e 4)$ & 80.6 & 31 & 0 & 24 \\
\hline $\operatorname{crtl}(e 5)$ & 95.8 & 24 & 0 & 27 \\
\hline $\operatorname{crtB}(e 6)$ & 92.5 & 27 & 0 & 29 \\
\hline crtYe(e7) & 93.3 & 45 & 0 & 24 \\
\hline $\operatorname{crtB}(e 8)$ & 95.8 & 24 & 0 & 23 \\
\hline crtYe(e9) & 95.6 & 23 & 0 & 24 \\
\hline $\operatorname{crtl}(\mathrm{e} 10)$ & 100 & 24 & 0 & 24 \\
\hline $\operatorname{crtl}(\mathrm{e} 11)$ & 95.8 & 24 & 0 & 27 \\
\hline $\operatorname{crtYf}(\mathrm{e} 12)$ & 100 & 27 & 0 & 29 \\
\hline $\operatorname{crtl}(e 13)$ & 100 & 29 & 0 & 31 \\
\hline $\operatorname{crtl}(\mathrm{e} 14)$ & 100 & 31 & 0 & 23 \\
\hline $\operatorname{crtl}(\mathrm{e} 15)$ & 95.6 & 23 & 0 & 36 \\
\hline $\operatorname{crtEb}\left(e_{16}\right)$ & 94.4 & 36 & 0 & 30 \\
\hline$c r t E b(e 17)$ & 76.6 & 30 & 0 & 31 \\
\hline $\operatorname{crtYf}(\mathrm{e} 18)$ & 80.6 & 31 & 0 & 24 \\
\hline crtEb(e19) & 95.8 & 24 & 0 & 27 \\
\hline crtYe(e20) & 92.5 & 27 & 0 & 45 \\
\hline $\operatorname{crtl}(e 21)$ & 93.3 & 45 & 0 & 24 \\
\hline crtYe(e22) & 95.8 & 24 & 0 & 23 \\
\hline $\operatorname{crtl}(e 23)$ & 95.6 & 23 & 0 & 24 \\
\hline$m 24$ & 100 & 24 & 0 & 24 \\
\hline$m 25$ & 95.8 & 24 & 0 & 27 \\
\hline$m 26$ & 100 & 27 & 0 & 29 \\
\hline$m 27$ & 100 & 29 & 0 & 24 \\
\hline$m 28$ & 100 & 24 & 0 & 27 \\
\hline$m 29$ & 96.2 & 27 & 0 & 23 \\
\hline$m 30$ & 95.6 & 23 & 0 & 24 \\
\hline
\end{tabular}




\begin{tabular}{|c|c|c|c|c|}
\hline$m 31$ & 95.6 & 23 & 0 & 24 \\
\hline$m 32$ & 94.4 & 36 & 0 & 27 \\
\hline$m 33$ & 76.6 & 30 & 0 & 29 \\
\hline m34 & 80.6 & 31 & 0 & 24 \\
\hline$m 35$ & 95.8 & 24 & 0 & 27 \\
\hline$m 36$ & 92.5 & 27 & 0 & 23 \\
\hline$m 37$ & 93.3 & 45 & 0 & 23 \\
\hline$m 38$ & 95.8 & 24 & 0 & 24 \\
\hline$m 39$ & 95.6 & 23 & 0 & 24 \\
\hline$m 40$ & 100 & 24 & 0 & 27 \\
\hline$m 41$ & 95.8 & 24 & 0 & 29 \\
\hline$m 42$ & 100 & 27 & 0 & 24 \\
\hline$m 43$ & 100 & 29 & 0 & 27 \\
\hline$m 44$ & 95.6 & 23 & 0 & 23 \\
\hline$m 45$ & 95.6 & 23 & 0 & 27 \\
\hline$m 46$ & 94.4 & 36 & 0 & 24 \\
\hline$m 47$ & 76.6 & 30 & 0 & 23 \\
\hline$m 48$ & 80.6 & 31 & 0 & 24 \\
\hline$m 49$ & 95.8 & 24 & 0 & 24 \\
\hline$m 50$ & 92.5 & 27 & 0 & 27 \\
\hline$m 51$ & 93.3 & 45 & 0 & 29 \\
\hline$m 52$ & 95.8 & 24 & 0 & 31 \\
\hline$m 53$ & 95.6 & 23 & 0 & 23 \\
\hline$m 54$ & 100 & 24 & 0 & 36 \\
\hline$m 55$ & 95.8 & 24 & 0 & 24 \\
\hline$m 56$ & 100 & 27 & 0 & 23 \\
\hline$m 57$ & 100 & 29 & 0 & 24 \\
\hline$m 58$ & 95.8 & 24 & 0 & 24 \\
\hline$m 59$ & 95.6 & 23 & 0 & 27 \\
\hline$m 60$ & 94.4 & 36 & 0 & 29 \\
\hline$m 61$ & 76.6 & 30 & 0 & 31 \\
\hline$m 62$ & 80.6 & 31 & 0 & 23 \\
\hline$m 63$ & 95.8 & 24 & 0 & 36 \\
\hline$m 64$ & 92.5 & 27 & 0 & 30 \\
\hline$m 65$ & 93.3 & 45 & 0 & 31 \\
\hline$m 66$ & 95.8 & 24 & 0 & 24 \\
\hline$m 67$ & 95.6 & 23 & 0 & 27 \\
\hline
\end{tabular}




\begin{tabular}{|c|c|c|c|c|}
\hline$m 68$ & 100 & 24 & 0 & 45 \\
\hline$m 69$ & 100 & 29 & 0 & 24 \\
\hline$m 70$ & 95.8 & 24 & 0 & 23 \\
\hline$m 71$ & 95.6 & 23 & 0 & 24 \\
\hline$m 72$ & 0 & 29 & 0 & 24 \\
\hline$m 73$ & 0 & 23 & 0 & 27 \\
\hline$m 74$ & 4.3 & 23 & 0 & 29 \\
\hline$m 75$ & 3.3 & 30 & 0 & 24 \\
\hline$m 76$ & 3.2 & 31 & 0 & 24 \\
\hline$m 77$ & 0 & 24 & 0 & 23 \\
\hline$m 78$ & 0 & 27 & 0 & 24 \\
\hline$m 79$ & 2.2 & 45 & 0 & 24 \\
\hline$m 80$ & 0 & 24 & 0 & 27 \\
\hline$m 81$ & 0 & 30 & 0 & 29 \\
\hline$m 82$ & 0 & 31 & 0 & 31 \\
\hline$m 83$ & 0 & 24 & 0 & 23 \\
\hline$m 84$ & 0 & 27 & 0 & 36 \\
\hline$m 85$ & 0 & 45 & 0 & 30 \\
\hline$m 86$ & 0 & 23 & 0 & 31 \\
\hline$m 87$ & 0 & 23 & 0 & 24 \\
\hline$m 88$ & 3.3 & 30 & 0 & 27 \\
\hline$m 89$ & 0 & 31 & 0 & 45 \\
\hline$m 90$ & 0 & 24 & 0 & 24 \\
\hline$m 91$ & 0 & 27 & 0 & 24 \\
\hline$m 92$ & 0 & 45 & 0 & 23 \\
\hline$m 93$ & 0 & 24 & 0 & 24 \\
\hline$m 94$ & 0 & 30 & 0 & 24 \\
\hline$m 95$ & 0 & 29 & 0 & 27 \\
\hline$m 96$ & 3.7 & 27 & 0 & 29 \\
\hline
\end{tabular}

